University of Nebraska - Lincoln

DigitalCommons@University of Nebraska - Lincoln

$1-29-2014$

\title{
Conflicting Selection in the Course of Adaptive Diversification: The Interplay between Mutualism and Intraspecific Competition
}

Rafael L.G. Raimundo

Jean P. Gibert

David H. Hembry

Paulo R. Guimaraes Jr.

Follow this and additional works at: https://digitalcommons.unl.edu/bioscifacpub

Part of the Biology Commons

This Article is brought to you for free and open access by the Papers in the Biological Sciences at

DigitalCommons@University of Nebraska - Lincoln. It has been accepted for inclusion in Faculty Publications in the Biological Sciences by an authorized administrator of DigitalCommons@University of Nebraska - Lincoln. 


\title{
Conflicting Selection in the Course of Adaptive Diversification: The Interplay between Mutualism and Intraspecific Competition
}

\author{
Rafael L. G. Raimundo, ${ }^{1}$ Jean P. Gibert, ${ }^{2}$ David H. Hembry, ${ }^{3, *}$ and Paulo R. Guimarães Jr. ${ }^{1, \dagger}$ \\ 1. Departamento de Ecologia, Instituto de Biociências, Universidade de São Paulo, Rua do Matão, Travessa 14, no. 321, 05508-900 Sao \\ Paulo SP, Brazil; 2. School of Biological Sciences, University of Nebraska, Lincoln, Nebraska 68588; 3. Department of Environmental \\ Science, Policy, and Management, University of California, Berkeley, California 94720
}

Submitted March 19, 2013; Accepted September 27, 2013; Electronically published January 29, 2014

Online enhancement: appendix.

AвSTRACT: Adaptive speciation can occur when a population undergoes assortative mating and disruptive selection caused by frequency-dependent intraspecific competition. However, other interactions, such as mutualisms based on trait matching, may generate conflicting selective pressures that constrain species diversification. We used individual-based simulations to explore how different types of mutualism affect adaptive diversification. A magic trait was assumed to simultaneously mediate mate choice, intraspecific competition, and mutualisms. In scenarios of intimate, specialized mutualisms, individuals interact with one or few individual mutualistic partners, and diversification is constrained only if the mutualism is obligate. In other scenarios, increasing numbers of different partners per individual limit diversification by generating stabilizing selection. Stabilizing selection emerges from the greater likelihood of trait mismatches for rare, extreme phenotypes than for common intermediate phenotypes. Constraints on diversification imposed by increased numbers of partners decrease if the trait matching degree has smaller positive effects on fitness. These results hold after the relaxation of various assumptions. When trait matching matters, mutualism-generated stabilizing selection would thus often constrain diversification in obligate mutualisms, such as ant-myrmecophyte associations, and in low-intimacy mutualisms, including plant-seed disperser systems. Hence, different processes, such as trait convergence favoring the incorporation of nonrelated species, are needed to explain the higher richness of low-intimacy assemblages_-shown here to be up to 1 order of magnitude richer than high-intimacy systems.

Keywords: assortative mating, disruptive selection, magic traits, trait complementarity, stabilizing selection.

\footnotetext{
* Current address: Center for Ecological Research, Kyoto University, 2-509-

3 Hirano, Otsu, Shiga 520-2113, Japan.

† Corresponding author; e-mail: prguima@usp.br.

Am. Nat. 2014. Vol. 183, pp. 363-375. (C) 2014 by The University of Chicago. 0003-0147/2014/18303-54551\$15.00. All rights reserved.

DOI: $10.1086 / 674965$
}

\section{Introduction}

Frequency-dependent intraspecific competition can generate disruptive selection within a population, favoring rare extreme phenotypes and promoting sympatric speciation (Rosenzweig 1978). Speciation as a result of intraspecific competition for resources also depends on assortative mating (in which individuals mate preferentially with mates similar to themselves) within ecologically diverging subpopulations (Dieckmann et al. 2004). The notion that disruptive selection caused by frequency-dependent competition can lead to speciation if associated with nonrandom mating has been formalized under the theoretical framework of adaptive diversification (Doebeli 2011). Studies on wild populations support critical assumptions of adaptive diversification models, such as the commonness of disruptive selection (Kingsolver et al. 2001; Bolnick and Fitzpatrick 2007) and of traits that are under natural selection and that also affect mate choice ("magic traits"; Servedio et al. 2011). On the other hand, adaptive diversification can often be constrained by conflicting forces, such as stabilizing selection caused by biotic interactions (Thompson 2005). Investigating scenarios of conflicting selection is thus a further step to deepen our understanding of how different types of ecological interactions shape adaptive diversification (Doebeli and Dieckmann 2000).

Organisms participate in several different types of interspecific interactions. These interactions exert potentially conflicting selective pressures, and their joint impact may shape diversification (Siepielski and Benkman 2009). Indeed, opposing selective pressures on the same trait exerted by different types of ecological interactions may be a ubiquitous phenomenon shaping phenotypic evolution and diversification (Strauss and Irwin 2004). The development of integrative frameworks exploring the interplay between 
intra- and interspecific ecological interactions is required to improve our understanding of how basic attributes of ecological interactions are connected with patterns of diversification at the community level (Fontaine et al. 2011; Jones et al. 2012). Mutualisms based on trait complementarity - that is, the functional matching between interacting species, such as the match between floral corolla depth and the length of hummingbird bills (Dalsgaard et al. 2008) - are examples of interspecific interactions that generate stabilizing selection and could counterweigh the diversifying effects imposed by intraspecific competition. Stabilizing selection emerges from trait complementarity because common intermediate phenotypes benefit more from mutualisms than do rare extreme phenotypes; the former are more likely to complement the most common local phenotypes of the other species (Thompson 2005). It is expected that this stabilizing effect will not be homogenous among different types of mutualisms because the wide variation in the natural history of mutualistic interactions should be associated with variable levels of dependence and species specificity (Bronstein 2009).

Indeed, different types of mutualisms vary in basic attributes that may affect diversification regimes. One of such attributes is the interaction intimacy, which is characterized by the degree of physiological integration and by the levels of physical and trophic dependence between the interacting species (Ollerton 2006; Fontaine et al. 2011). Symbiotic mutualisms are extreme cases of high-intimacy interactions in which individuals of one species (the symbiont) have an extensive physical and physiological integration with another species (the host), as exemplified by plant-Rhizobium associations (Bronstein 2009). As a consequence of a tight biological integration, individuals in high-intimacy systems tend to interact with few individuals of the other species during their lifetimes (Guimarães et al. 2007; Pires and Guimarães 2013). In contrast, seed dispersal systems illustrate low-intimacy mutualisms in which individuals of the interacting species have a low degree of biological integration and physical dependence (Ollerton 2006). Organisms involved in low-intimacy mutualisms may interact with many interspecific individuals during their lifetimes, as occur in interactions between plants with diverse seed dispersal strategies and generalist seed dispersers (Herrera 1995). Although interaction intimacy at the individual level is not necessarily correlated to specialization at the species level, there are well-known cases in which high interaction intimacy is correlated to high specialization, as occur in mutualisms between sea anemones and associated species of anemonefishes (Ricciardi et al. 2010). Conversely, low interaction intimacy at the individual level is often correlated to low specialization at the species level, as illustrated by generalized pollinators and generalist-pollinated plants (Waser 1996).
Another feature of mutualistic interactions that can affect diversification regimes is the extent to which trait matching affects fitness. In nature, the degree of the positive impact of trait matching on fitness is highly variable. For instance, in organisms involved in intimate associations, mismatched individuals that are unable to perform mutualistic interactions may even be excluded from the mating pool, as occurs in associations between ants and myrmecophytes (Fonseca and Ganade 1996; Fonseca 1999). Similarly, the fitness consequences of mutualistic interactions between self-incompatible plants and specialized pollinators may be highly dependent on trait complementarity (Anderson and Johnson 2008; Thompson et al. 2013). However, phenotype matching may be less important in low-intimacy interactions between species involved in interspecific cooperative hunting (Minta et al. 1992; Bshary et al. 2006).

Here, we used individual-based simulations to assess how the interplay between mutualistic interactions and intraspecific competition affects adaptive diversification. We focused on mutualisms in which trait complementary between the interacting species plays a role in the fitness outcomes of the interaction (Bascompte and Jordano 2007). In nature, reciprocal fitness benefits commonly favors trait complementarity in various mutualistic systems, from pollination to plant protection by ants (Thompson 2005; Anderson and Johnson 2008; but see Anderson et al. 2010 for different mechanisms resulting in trait mismatches between mutualistic species). We thus incorporated mutualisms characterized by trait complementarity into a scenario of adaptive speciation. In doing so, we assumed that a magic trait mediates mate choice and intraspecific competition and also affects mutualistic interactions (Bhattacharyay and Drossel 2005). For example, body size works as a magic trait in various taxa (Servedio et al. 2011) and is often involved in mutualistic trait complementarity, as exemplified by fitness advantages to ant queens whose body sizes match the sizes of available domatias of myrmecophytes (Fonseca 1999). Furthermore, two or more genetically correlated traits, one involved in divergent selection and other in assortative mating, can work as a magic phenotype that also mediates mutualistic interactions. For example, in Darwin finches (Geospiza spp.), beak morphology (which affects song patterns) and body size are considered a magic phenotype (Servedio et al. 2011). The same traits may mediate mutualisms in at least seven sympatric Geospiza species that are known to act as effective seed dispersers (Guerrero and Tye 2009).

We designed two simulation experiments to investigate how different types of ecological interactions at the individual level affect the diversification patterns observed at the species level. These simulation experiments encompass distinct scenarios for the interplay between the di- 
versifying effects generated by intraspecific competition and the stabilizing effects of mutualisms. In a first simulation experiment, we investigated species diversification in the case of an intimate, specialized association in which individuals have few mutualistic partners and the interaction has a high impact on fitness. Specifically, we explored the effects on diversification imposed by the fates of individuals that remain without any mutualistic partner. We contrasted simulations in which individuals unable to perform mutualistic interactions persist in the mating pool (as in nonobligate mutualisms) to simulations in which they are removed from the population and thus are unable to reproduce (as in obligate mutualisms). In a second simulation experiment, we explored the effects imposed by different types of mutualisms on diversification. To simulate different types of mutualisms, we varied two interaction attributes associated to the interaction intimacy: (i) the number of different individual partners with which individuals interact and (ii) the effect of the degree of phenotype matching on fitness.

\section{The Model}

We used individual-based models (IBMs) to simulate phenotypic evolution in a pair of free-living species, $A$ and $B$, which are reciprocally involved in mutualistic interactions. Both species reproduce sexually in discrete, nonoverlapping generations. The individuals of each species are characterized by magic traits $z_{\mathrm{A}}$ and $z_{B}$, which mediate intraand interspecific ecological interactions and influence mate choice. The initial values of $z_{A}$ and $z_{B}$ are assigned by sampling from Gaussian distributions with means $\bar{z}_{A}$ and $\bar{z}_{B}$ and standard deviations $\sigma_{A}$ and $\sigma_{B}$, respectively. During a given time step, individuals (i) face environmental stabilizing selection (which excludes selective pressures imposed by competition and mutualism), (ii) experience intraspecific competition, (iii) engage in mutualistic interactions, (iv) reproduce, and die. We defined $N_{A}$ as the number of individuals in species $A$ or of all species derived from $A$ and $N_{B}$ as the number of individuals of species $B$ or of all species derived from $B$. The same numbers of individuals $N_{A}=N_{B}$ are born at each time step. To simulate populations at the carrying capacity, $N_{A}$ and $N_{B}$ are constant over time, implying intraspecific competition is relevant to ecological dynamics. We later relax these assumptions. Further details are given below.

\section{Stabilizing Selection}

Stabilizing selection accounts for all selective pressures on traits $z_{A}$ and $z_{B}$ imposed by the environment, excluding those exerted by intraspecific competition and mutualism. Any given individual $i$ of a species $K$ (with $K$ being $A$, $B$, or the species originating from $A$ or $B$ ) displaying the phenotype $z_{K_{i}}$ has a survival probability $P_{\text {surv }}\left(z_{K_{i}}\right)$, defined as

$$
P_{\text {surv }}\left(z_{K_{i}}\right)=\exp \left[-\gamma\left(z_{K_{i}}-\theta_{K}\right)^{2}\right]
$$

where $\theta_{K}$ is the phenotype of species $K$ favored by stabilizing selection and $\gamma$ is degree of sensitivity of that individual to stabilizing selection, which is equal for $A$ and $B$. For a fixed value of $z_{K_{i}}$ as $\gamma$ increases, $P_{\text {surv }}\left(z_{K_{i}}\right)$ decreases from 1 (no selection) to approximately 0 (strong selection). To simulate environment-induced mortality, we randomly assign a number, $r_{z_{K_{i}}}$, sampled from a uniform distribution between 0 and 1 , to each individual $i$. Individual $i$ dies if $r_{z_{K_{i}}}>P_{\text {surv }}\left(z_{K_{i}}\right)$ (Yoder and Nuismer 2010).

\section{Intraspecific Competition}

We assume that conspecific individuals with similar phenotypes $\left(z_{K_{i}}=z_{K_{j}}\right)$ compete more intensively than do more dissimilar individuals. Consequently, competition among individuals for resources that are extrinsic to the mutualistic interaction results in frequency-dependent disruptive selection. The function describing the competition between two individuals of species $K$ with phenotypes $z_{K_{i}}$ and $z_{K_{j}}$ is

$$
\alpha\left(z_{K_{i}}, z_{K_{j}}\right)=\exp \left[-c\left(z_{K_{i}}-z_{K_{j}}\right)^{2}\right],
$$

such that higher $c$ is associated with stronger competition (Bürger et al. 2006). The function $P_{\text {comp }}$ describes the effect of frequency-dependent intraspecific competition on individuals' mating probabilities. We assume that competition linearly reduces $P_{\text {comp. }}$. Furthermore, in our baseline model, we assumed that the fitness components arising from ecological interactions affect only females mating probabilities (see the section "Relaxation of Assumptions" below for the alternative scenario in which ecological interactions also affects the males mating probabilities). The effect of competition on a female $i$ of a species $K$ is defined by

$$
P_{\text {comp }}\left(z_{K_{i}}\right)=\frac{1-\bar{\alpha}\left(z_{K_{i}}\right)}{\max _{p}\left(1-\bar{\alpha}\left(z_{K_{p}}\right)\right)},
$$

where $p=1,2, \ldots, N_{K_{f}}$ Here, $\bar{\alpha}\left(z_{K_{i}}\right)$ is the average intraspecific competition faced by an individual $i$ with phenotype $z_{K_{i}}$ with respect to all other individuals within the population. Term $N_{K_{\mathrm{f}}}$ is the number of females within the population of the species $K$. The denominator of equation (3) rescales the values of $P_{\text {comp }}\left(z_{K_{i}}\right)$ to the largest degree of intraspecific competition endured by females in the population, thus providing a proxy for the relative female fitness component arising from intraspecific competition. An equivalent relative fitness component can be computed for males in a similar way. In the baseline model, we assume that both species experience intraspecific competition. We later relax this assumption. 


\section{Mutualisms}

Individuals of species $B$ (or of the species derived from $B$ ) evaluate and select mutualistic partners of species $A$ (or of the species derived from $A$ ), simulating common interactions between organisms that actively choose mutualistic partners (e.g., animals) and organisms being chosen (e.g., plants; Bronstein et al. 2006). Interactions are based on trait complementarity. For simplicity, we assume that higher complementarity occurs when $z_{A_{i}}=z_{B_{i}}$. Every individual $i$ of species $B$ interacts with a fixed number $\omega_{B_{i}}$ of individuals from species $A$ bearing the most complementary traits. Therefore, the number $\omega_{A_{i}}$ of different partners with which each individual $i$ of species $A$ interacts is variable and defined by the attractiveness of its phenotype $\left(z_{A_{i}}\right)$ to individuals of species $B$ (Gómez and Perfectti 2012). The fitness component arising from the benefits gained from the mutualistic interactions for a female $i$ of species $A$ with phenotype $z_{A_{i}}$, $P_{\text {mut }}\left(z_{A_{i}}\right)$, depends on the degree of trait complementarity between that female and the number $\omega_{A_{i}}$ of interacting interspecific individuals it encounters during its life span, as defined by the following equation:

$$
P_{\mathrm{mut}}\left(z_{A_{i}}\right)=\left(\frac{1+\sum_{j=1}^{N_{B}} \rho_{i j} \exp \left[-\left(z_{A_{i}}-z_{B_{j}}\right)^{2}\right]}{\max _{p}\left\{1+\sum_{q=1}^{N_{B}} \rho_{p q} \exp \left[-\left(z_{A_{p}}-z_{B_{q}}\right)^{2}\right]\right\}}\right)^{\lambda},
$$

with $p=1,2, \ldots, N_{A_{f}}$ and in which $\rho_{i j}=1$ if individual $i$ interacts with individual $j$ or 0 otherwise. The number of interspecific individuals interacting with the female $i$ of the species $A$ is thus $\omega_{A_{i}}=\sum_{j=1}^{N_{B}} \rho_{i j}$. Term $N_{A_{\mathrm{f}}}$ is the number of females within the population of the species $A$. Here the denominator rescales the values of $P_{\text {mut }}$, hence providing a proxy for the relative female fitness component arising from mutualistic interactions. The parameter $\lambda$ measures the effect of the degree of phenotype matching on fitness. The negative effect of increased trait mismatching on fitness thus increases as $\lambda$ increases. We assumed that $\lambda$ varies between 0 and 1 . We used $\lambda=1$ as the maximal effect of phenotypic matching on fitness. In this case, equation (4) is similar to the equation describing the fitness consequences of phenotypic matching used in previous works (e.g., Yoder and Nuismer 2010). When $0<\lambda$ $<1$, mismatched interactions are progressively less penalized under decreasing values of $\lambda$ (appendix, fig. A1; appendix and figs. A1-A13 available online). If $\lambda=0$, mutualism has no impact on the mating probability of individuals. The reproductive outcome of mutualistic interactions for a female $i$ of species $B$ is computed according to the same scheme.

\section{Mate Choice, Reproduction, and Death}

Each female of species $K$ (with $K$ being species $A$ or $B$ or the species derived from them) with phenotype $z_{K_{i}}$ has a mating probability $P_{\text {mat }}\left(z_{K_{i}}\right)$, determined by the reproductive penalty imposed by intraspecific competition $P_{\text {comp }}\left(z_{K_{i}}\right)$ and the reproductive outcome of the mutualistic interaction, $P_{\text {mut }}\left(z_{K_{i}}\right)$, as defined by

$$
P_{\text {mat }}\left(z_{K_{i}}\right)=\frac{P_{\text {comp }}\left(z_{K_{i}}\right) \times P_{\text {mut }}\left(z_{K_{i}}\right)}{\sum_{j=1}^{N_{K_{f}}} P_{\text {comp }}\left(z_{K_{j}}\right) \times P_{\text {mut }}\left(z_{K_{j}}\right)} .
$$

For each offspring, a parental female is sampled with replacement with a probability given by $P_{\text {mat }}\left(z_{K_{i}}\right)$. That female mates with the male bearing the most similar trait value. The mating pair generates a single offspring individual with a trait value defined by the mean of the parental trait values plus or minus segregation variance, modeled as a random number sampled from a Gaussian distribution with mean $\bar{V}_{A}=0$ and standard deviation $\sigma_{V_{A}}=0.05$. All individuals die after reproducing, and their offspring become the next generation.

\section{Number of Different Mutualistic Partners and Impact of Trait Matching on Fitness}

Models of mutualisms often assume that only a single pairwise interaction between individuals occurs. Nevertheless, a variable number of individual partners may have important effects on the ecological and evolutionary dynamics of mutualisms (Guimarães et al. 2007; Gokhale and Traulsen 2012). We thus investigated the effect of partner number on adaptive diversification by varying the number of different individual mutualistic partners $\left(\omega_{B}\right)$ for each individual $i$ of species $B$. Intimate mutualisms, such as those between ants and myrmecophytes, are cases in which individuals (a plant or ant colony) have low $\omega_{B}$. In contrast, low-intimacy mutualists, such as nectarivorous insect pollinators, have large $\omega_{B}$. One could expect individuals to interact with the same optimal partner multiple times because this partner provides the highest fitness benefit. However, in mutualisms among free-living species, individuals interact with multiple partners due to interspecific competition (Palmer et al. 2003), high temporal variation in partner availability (Tarayre 2007), increased predation risk when visiting the most rewarding partners (Jones 2010), and reward variability among partners, such as interindividual variability in amino acid composition of nectar within plant populations (Lanza et al. 1995).

In nature, the number of partners with which an individual interacts is variable. However, to test the effects of the number of interacting partners in a controlled way, we assumed that $\omega_{B}$ is fixed, meaning that all individuals of species $B$ interact with the same number of different 
mutualistic partners. On the other hand, individuals of species $A$ have no constraints on the number of different partners with which they can interact; therefore, $\omega_{A}$ depends on the choice patterns of the individuals of species $B$. This difference implies an asymmetry between species regarding the number of individual mutualistic partners per individual. Asymmetry in the number of interspecific partners occurs, for example, when plants produce abundant resources that can be used by many individual animals, but each animal interacts with only a few individual plants (Bentley 1977; Weiblen 2002). Complementarily, we examined the case in which individuals of both species have constraints on the total number of partners during their lifetimes (appendix).

We also investigated the consequences for adaptive diversification of having variable impacts of the mutualism trait matching on fitness. Varying $\lambda$ (eq. [4]) changes the effect of the degree of trait matching on the fitness component arising from the mutualistic interaction, thus affecting individuals' mating probabilities (fig. A1). High $\lambda$ values simulate scenarios in which the degree of trait matching strongly affects the reproductive output of an individual, with mismatched individuals being highly penalized, as in the case of self-incompatible plants and specialized pollinators (Anderson and Johnson 2008). At the other extreme, minimal $\lambda$ values simulate mutualisms in which the degree of trait matching has weak reproductive effects, as is likely the case for interspecific cooperative hunting, such as that between the badger Taxidea taxus and the coyote Canis latrans (Minta et al. 1992).

\section{Simulation Experiments}

We performed a sensitivity analysis (appendix) to identify the parameter combination resulting in greater species diversification. This parameter combination $\left(\sigma_{A}=\sigma_{B}=\right.$ $\left.0.58, \gamma=10^{-3}, c=0.74\right)$ defined the baseline scenario for two simulation experiments designed to explore the hypothesis that mutualism attributes constrain adaptive diversification. In our simulations, a species was heuristically defined as any phenotypic cluster including reproductively isolated individuals that is differentiated from other phenotypic clusters by a distance of at least 0.2 units of $z_{K}$. Comparisons of species richnesses among simulations were possible because the number of emerging species reached asymptotic levels before 1,000 time steps. See the appendix for further details on species delimitation.

\section{Experiment 1: Intimate Mutualisms, Mating Pool Composition, and Diversification}

In the first simulation experiment, we modeled an intimate specialized mutualism to investigate how alternative re- productive outcomes for individuals that fail to perform mutualistic interactions affect diversification. The first simulation group (group 1: control) modeled evolution in the absence of mutualism $\left(\omega_{B}=\lambda=0\right)$, with individual mating probabilities depending exclusively on $P_{\text {comp. }}$. In groups 2 and 3, species coevolve through mutualism, defined by $\omega_{B}=\lambda=1$. Therefore, in groups 2 and 3 , each individual of species $B$ interacts with only one mutualistic partner, and the degree of trait matching has a strong impact on its reproductive output. Individuals of species $A$ may interact with an unrestricted number of interspecific individuals $\left(\omega_{A}\right)$. However, for low $\omega_{B}$, some individuals of species $A$ will not have any mutualistic interactions (appendix). Groups 2 and 3 exhibit contrasting potential reproductive consequences for individuals of species $A$ that do not have any mutualistic interactions. In group 2, individuals without mutualistic partners are able to reproduce (i.e., the mutualism is not obligate). For individuals without mutualistic partners, the numerator of equation (4) is equal to 1 , since in this case the summation of mutualistic trait matching effects is equal to 0 . In group 3 , individuals without mutualistic interactions do not survive to mate (i.e., the mutualism is obligate). We ran 50 simulation replicates in each group with $\sigma_{A}=\sigma_{B}=0.58$, $\gamma=10^{-3}, \theta_{A}=0, \theta_{B}=1, c=0.74$, and $\lambda=1$. Pairwise differences in the mean asymptotic richness between these groups were tested using Tukey's HSD test.

The asymptotic species richnesses of control simulations (group 1: $\omega_{B}=0, \bar{S}_{A}=7.5 \pm 1.1$ species; $\bar{S}_{B}=7.3 \pm$ 0.94 species) are not significantly different from the richnesses observed in simulations of nonobligate mutualisms (group 2), in which individuals that fail to engage in interspecific interactions remain in the mating pool $\left(\omega_{B}=\right.$ $1, \bar{S}_{A}=7.2 \pm 0.92$ species; $\bar{S}_{B}=7.05 \pm 1.05$ species; Tukey's HSD test, $Q=2.36, P>.050$ for $S_{A}$ and $S_{B}, n=$ 150 simulations). However, when individuals of species $A$ that fail to engage in mutualistic interactions are not permitted to mate (obligate mutualisms, group 3), the mean asymptotic species richnesses are significantly lower than those of control simulations $\left(\omega_{B}=1, \bar{S}_{A}=6.75 \pm 0.91\right.$ species; $\bar{S}_{B}=6.60 \pm 0.83$ species; Tukey's HSD test, $Q=$ 2.36, $P<.001, n=150$ simulations).

\section{Experiment 2: The Effect of Different Types of Mutualism on Diversification}

To explore the effects of different types of mutualism on diversification, we simulated 30 scenarios (with 20 replicates for each) defined by the number of different individual partners, $\omega_{B}(1,2,4,8,16,32,64,128,256$, and $512)$ and by the magnitude of the impact of trait matching on fitness, $\lambda(0.01,0.1$, and 1$)$. Since the maximum value of $\omega_{B}$ is relatively low, we assumed that, for the range of 
$\omega_{B}$ values considered here, the net benefits of mutualism do not saturate under increased number of partners. Preliminary analyses showed that for $\omega_{B}>4$ all individuals of species $A$ interact (appendix, fig. A7). We investigated the scenario in which individuals of species $A$ without mutualistic partners are able to mate (as in group 2 in experiment 1), controlling the potential confounding effect of removing individuals from mating pool in simulations in which $\omega_{B}<4$ (experiment 1 ). To control for the effects of mutualisms on diversification, we ran 20 additional simulations without mutualistic interactions $\left(\omega_{B}=\lambda=\right.$ $0)$. In each replicate, we counted the asymptotic richness of species derived from $A\left(S_{A}\right)$ and $B\left(S_{B}\right)$ after 1,000 time steps. We tested the effects of $\omega_{B}$ and $\lambda$ on diversification using general linear models (GLMs) in which the values of $\omega_{B}$ and $\lambda$ were log transformed to improve normality and homoscedasticity.

Both the number of individual mutualistic partners $\left(\omega_{B}\right)$ and the effect of the degree of trait matching on fitness $(\lambda)$ affect species richness emerging from adaptive diversification (figs. 1, 2). Richness is inversely correlated with the number of different individual partners, $\omega_{B}$. Indeed, $S_{A}\left(\mathrm{GLM}, F_{2,617}=322.4, P<.001, n=620\right)$ and $S_{\mathrm{B}}$ (GLM, $\left.F_{2,617}=263.4, P<.001, n=620\right)$ decrease as individuals of species $B$ interact with a greater number of mutualistic partners (i.e., with increasing $\omega_{B}$ values). An increased impact of the degree of trait matching on fitness (increasing $\lambda$ ) also constrains species diversification (GLM, $F_{2,617}=185.7$ and $F_{2,617}=309.9$, for $S_{A}$ and $S_{B}$, respec- tively; $P<.001, n=620$; fig. 2 ). When trait matching has a strong impact on the mating probabilities of individuals $(\lambda=1)$, a higher number of different individual mutualistic partners $\left(\omega_{B}\right)$ leads to decreased phenotypic variance (fig. 1) and also progressively constrains the emerging species richness (fig. $2 A$ ). A decrease in diversification with the increase in the number of individual mutualistic partners $\left(\omega_{B}\right)$ is also observed when the impact of trait matching on mating probabilities $(\lambda)$ is decreased by an order of magnitude (fig. $2 B$ ). Although species richness is lower when $\omega_{B} \geq 64$, extreme $\omega_{B}$ values do not impose additional constraints on species diversification at lower $\lambda$ values (fig. $2 B$ ). The effect of $\omega_{B}$ on richness disappears when $\lambda$ is very small (0.01) because the effect of the mutualism is not sufficient to impose the necessary stabilizing selection (fig. 2C).

\section{Relaxation of Assumptions}

We explored the dynamics of the model under the relaxation of different assumptions about mutualistic interactions, selection regimes, and population densities. Relaxing these assumptions allowed us to explore the dynamics of the IBM under alternative biological scenarios.

\section{Phenotype Matching}

In experiments 1 and 2, individuals of species $B$ always choose a number $\omega_{B}$ of mutualistic partners that represent
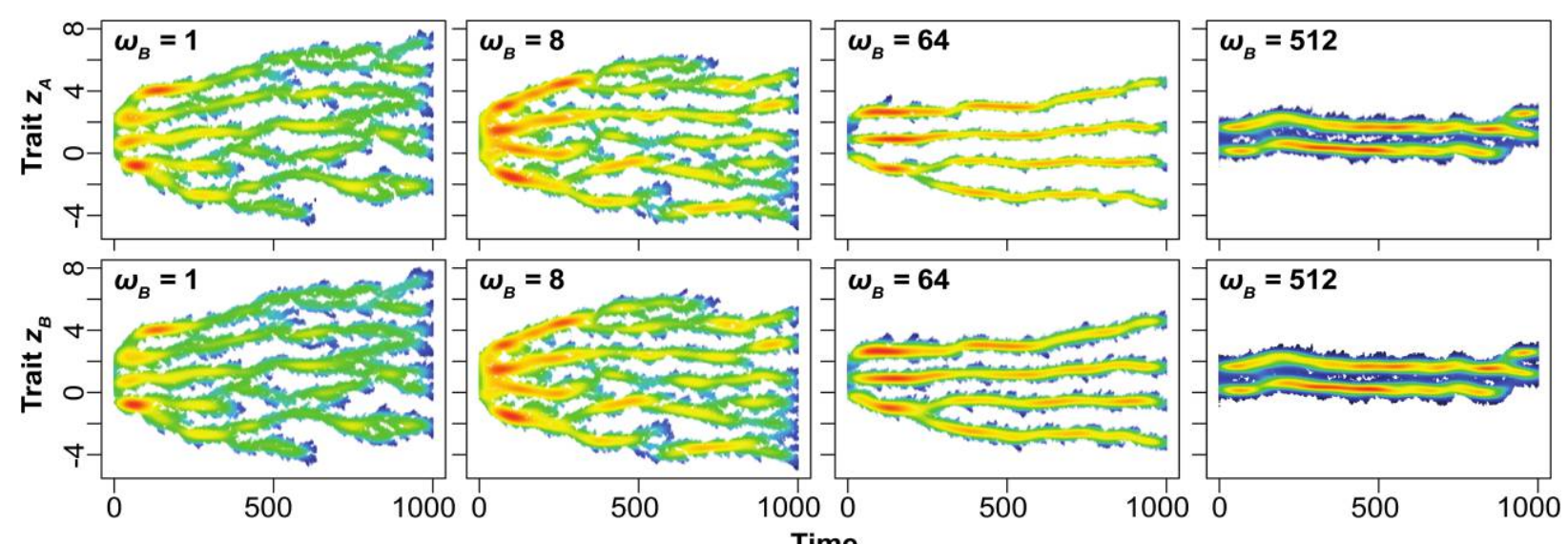

Figure 1: Mutualism attributes affect adaptive diversification. We consider sympatric populations of two mutualistic species, $A$ and $B$, both experiencing intraspecific, frequency-dependent competition for resources. Mutualistic interactions are based on phenotypic matching, and the same trait mediates intraspecific competition and female mate choice. Individuals of species $B$ choose a limited number $\left(\omega_{B}\right)$ of mutualistic partners, whereas individuals of species $A$ have no constraints on the number of different interspecific individuals with which they interact. Here, the impact of mutualism trait matching on fitness is high because $\lambda=1$ (see eq. [4]). Females mate assortatively, and the product of the penalty imposed by intraspecific competition and the profit gained from mutualism defines individual mating probabilities. The color temperature indicates the density of individuals, ranging from high (hot colors) to low (cool colors). $N_{A}=N_{B}=600, \sigma_{A}=\sigma_{B}=0.58$, $\gamma=10^{-3}, \theta_{\mathrm{A}}=0, \theta_{\mathrm{B}}=1, c=0.74$. 

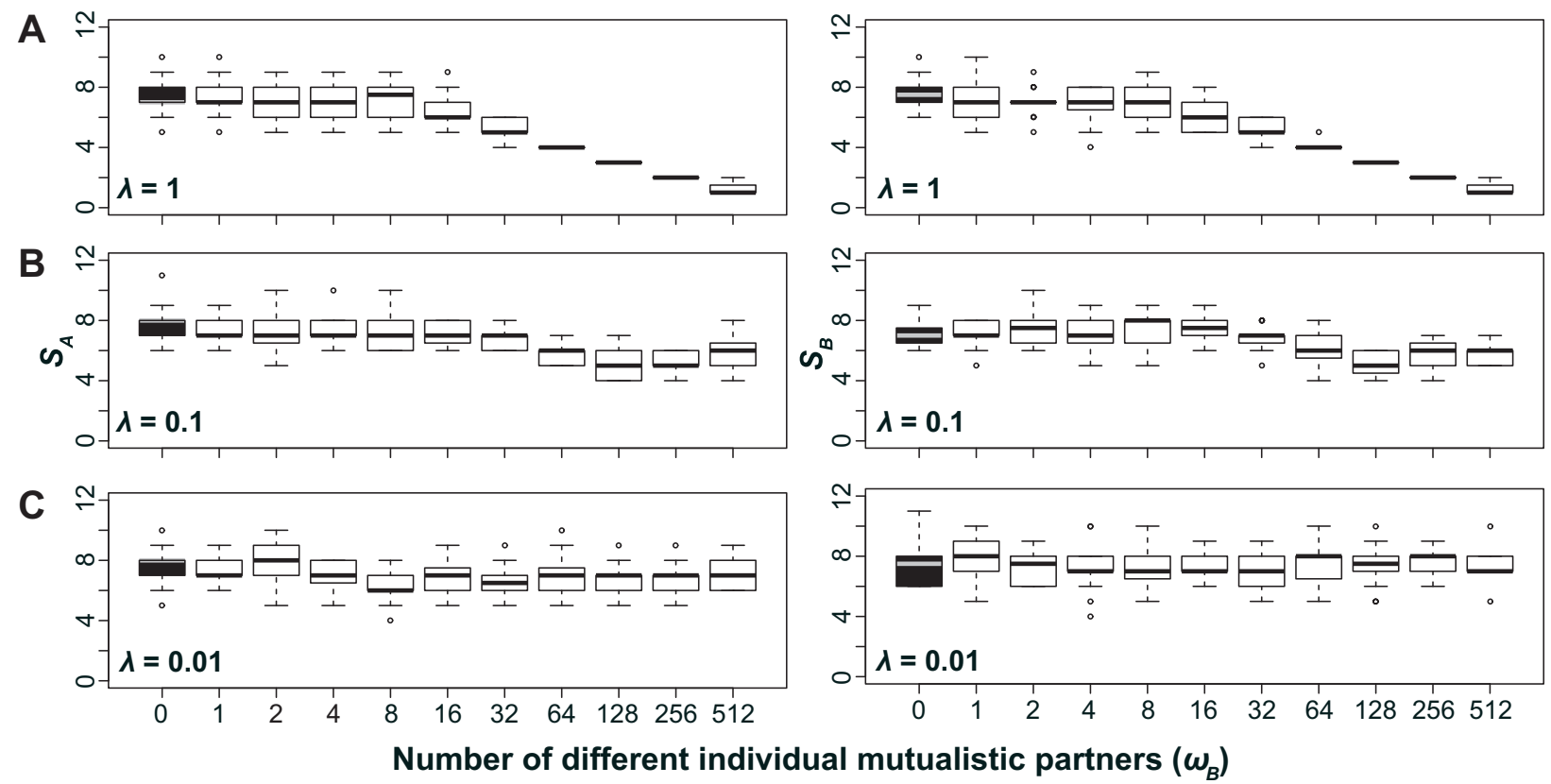

Figure 2: Species richnesses achieved in the simulation experiment under variable mutualisms. Asymptotic species richnesses obtained in simulations with different mutualism attributes, namely, the number of different interspecific partners of individuals of species $B\left(\omega_{B}\right)$ and the impact of mutualistic trait matching on the individual mating probabilities $(\lambda)$. Each panel shows species richnesses under variable $\omega_{B}$ values for mutualisms in which the degree of trait matching has a high impact on fitness $(A)$, has an intermediate impact on fitness $(B)$, or has a low impact on fitness $(C)$. Twenty replicate simulations were performed for each scenario. The horizontal lines inside the boxes indicate the medians. The boxes encompass the interquartile range, and error bars indicate the smallest (lower) and largest (upper) nonoutliers. Outliers are plotted separately as points.

the best trait matches in the entire population of species $A$. Therefore, individuals are assumed to be able to evaluate all existing interspecific individuals. We relaxed this unrealistic assumption by performing a simulation in which each individual $i$ of species $B$ randomly chooses its mutualistic partner(s) from a subset of the population of $A$ formed by individuals within a certain phenotype range. This range is defined for each individual $i$ of species $B$ as a percentage $\psi$ of individuals of species $A$ bearing the traits most similar to its trait $Z_{B:}$. We ran 20 replicates for each combination of four $\psi$ values $(15 \%, 30 \%, 45 \%, 60 \%)$ with four $\omega_{B}$ values $(1,8,32,64)$ and $\lambda=1$. We then used a GLM to estimate the effects of $\psi$ and $\omega_{B}$ in this set of simulations. This alternative phenotype-matching scheme leads to a more intense constraint on diversification due to mutualism than is observed in experiments 1 and 2. The lower the subset of potential partners $\psi$, the lower the final species richness (GLM, $F_{2,317}=135.2$ and $F_{2,317}=176.6$ for $\bar{S}_{A}$ and $\bar{S}_{B}$, respectively; $P<.001, n=$ $320)$. Nevertheless, the constraints imposed by the number of partners (GLM, $F_{2,317}=87$ and $F_{2,317}=80.4$ for $\bar{S}_{A}$ and $\bar{S}_{B}$, respectively; $P<.001, n=320$ ) still hold after relaxing the assumption of best-fit phenotype matching in our baseline model.

\section{$\mathrm{P}_{\text {mut }}$ Computation}

Similar constraining effects of increasing the number of mutualistic partners on diversification arise if the impact of mutualism on reproduction does not depend on the quantity of individual mutualistic partners but rather on how beneficial the interactions are on average, that is, if $P_{\text {mut }}$ is computed using the mean instead of the sum to aggregate the effect of interaction events in equation (4) (appendix).

\section{Males under Selective Pressures Arising from Ecological Interactions}

In our baseline model, only females have mating probabilities defined by two fitness components arising from ecological interactions, $P_{\text {comp }}$ and $P_{\text {mut }}$ (eqq. [3]-[5]). Therefore, for males, mating probabilities are determined exclusively by females' choice, since each breeding female chooses a male with the most similar trait value as its sexual partner. In order to investigate if our model results hold if males are also affected by the selective pressures generated by ecological interactions, we explored the effects of the number of individual mutualistic partners, $\omega_{B}$, 
and of the impact of the degree of trait matching on fitness, $\lambda$, when the male mating pool is formed by a percentage ( $50 \%$ or $25 \%$ ) of the original male subpopulation. The probability that a male will remain in the population to form the mating pool is computed following equations (3)-(5), but with $N_{A_{\mathrm{m}}}$ and $N_{K_{\mathrm{m}}}$, the number of males within the population of the species $A$ and $K$, replacing $N_{A_{\mathrm{f}}}$ and $N_{K_{\mathrm{f}}}$, respectively. We found that the effects of $\omega_{B}$, and $\lambda$ in these simulations in which ecological interactions also affect males' survival and in our baseline model are qualitatively the same (appendix).

\section{Both Species with Limited Numbers of Individual Mutualistic Partners}

We relaxed the assumption that only species $B$ has constraints on the number of mutualistic partners with which individuals interact. Increasing the number of interacting partners also reduces diversification if both species are constrained in the number interacting individuals $\left(\omega_{A}=\right.$ $\omega_{B}$; appendix).

\section{Only One Species under Disruptive Selection}

When we relaxed the assumption that both species are experiencing disruptive selection, similar effects of $\omega_{B}$ and $\lambda$ on diversification emerge when only species $B$ faces disruptive selection caused by intraspecific competition (appendix). However, diversification is even more constrained when species $A$, which is the one being chosen within the mutualistic interaction, experiences disruptive selection due to intraspecific competition (appendix).

\section{Asymmetric Population Sizes}

We explored the diversification dynamics when the population sizes of species $A$ and $B$ are asymmetric. We used values of $N_{B}<N_{A}$, leading to increased competition among individuals of species $A$ for mutualistic partners. We ran 20 replicates for three values of $N_{B}(75,150,300)$ and four $\omega_{B}$ values $(1,8,32,64)$ under $\lambda=1$ and $N_{A}=600$. The population size of species $B\left(N_{B}\right)$ affects the diversification of both species. Lower $N_{B}$ is associated with a lower final richness of species derived from species $B$ (GLM, $F_{2,237}=$ 168.3 and $F_{2,237}=327.7$ for $\bar{S}_{A}$ and $\bar{S}_{B}$, respectively; $P<$ $.001, n=240)$. This result is a consequence of stronger stabilizing selection imposed by the competition among individuals of $A$ for the fewer individuals of $B$. After controlling for the effect of $N_{B}$, the number of partners, $\omega_{B}$, still imposes constraints on diversification similar to those detected in experiment 2 (GLM, $F_{2,237}=187.1$ and $F_{2,237}=97.6$ for $\bar{S}_{A}$ and $\bar{S}_{B}$, respectively; $P<.001, n=$ 240).

\section{Stochastic Variation of Population Sizes}

To relax the assumption of constant population sizes, we modeled two different scenarios for population variation across time. In the first model, the overall numbers of individuals $N_{B}$ and $N_{A}$ varied independently and randomly around a mean. At each generation, the number of individuals is the initial population size $\left(N_{A}=N_{B}=600\right)$ summed to a parameter $e$ with a value sampled from a Gaussian distribution with a mean of 0 and a standard deviation of $\sigma_{P(K)}$. This stochastic variation in population size had a small but significant effect, constraining diversification (GLM, $F_{2,237}=5.7$ and $F_{2,237}=0.6$ for $\bar{S}_{A}$ and $\bar{S}_{B}$, respectively; $\left.P<.001, n=240\right)$. After controlling for the effect of population fluctuations, the number of mutualistic partners, $\omega_{B}$, still had an important effect of constraining diversification $\left(F_{2,237}=467.2\right.$ and $F_{2,237}=297.7$ for $\bar{S}_{A}$ and $\bar{S}_{B}$, respectively; $\left.P<.001, n=240\right)$. In a second scenario, we modeled more drastic variations in the overall number of individuals. From initial population sizes $N_{0(A)}=\mathrm{N}_{0(B)}=600$, the number of individuals $N_{t(K)}$ of a species at generation $t$ is $N_{t-1(K)}+V$, where $V$ is a percentage of $N_{t-1(K)}$. At any given generation, $V$ is randomly assigned to be positive or negative. We contrasted the diversification dynamics under two values of $\omega_{B}(8$, $32)$ and $V(1 \%, 5 \%)$. In the resulting population dynamics, population sizes are uncorrelated, independently assuming values much lower or much higher than the original sizes. Despite such severe variation in population sizes, the general trend of decreased species diversification following increasing $\omega_{B}$ persisted (appendix).

\section{Discussion}

The interplay between disruptive and stabilizing selection shapes the phenotypic variability of natural populations (Bürger 2002). These selective forces are thought to be equally common in nature (Kingsolver et al. 2001) and are likely to act together within populations (Felsenstein 1979). They thus have the potential to jointly affect the selective regimes underpinning the adaptive origin of new species. Indeed, conflicting selection pressures can affect the microevolutionary processes involved in phenotypic divergence and speciation (Lankinen and Larsson 2009). Our simulations indicate that, even under strong disruptive selection and assortative mating, mutualisms based on phenotype matching can constrain diversification in different ways.

First, mutualisms in which individuals have few partners can constrain diversification if the interaction determines which individuals are able to mate. Extreme phenotypes thus become less frequent in the mating pool even if they benefit from low intraspecific competition. Direct depen- 
dence on mutualism for reproduction occurs when species rely on interspecific interactions for mating, as in the case of self-incompatible plants in relation to pollinators (Bond 1994). Additionally, in obligate mutualisms, individuals that fail to interact with partners suffer severe reproductive consequences. For example, ant queens that are excluded in the competition for a limited number of myrmecophyte plants are unable to survive and reproduce (Fonseca 1999). In other cases, plant individuals with maladapted myrmecophilic traits are attacked by herbivores and fail to reach reproductive size due to a lack of protection by ants (Léotard et al. 2008). Our analyses show that the emerging species richness depends on the population sizes of interacting species. Indeed, larger population sizes increase rates of adaptive diversification (Stevens et al. 2007). We thus expect that the magnitude of the constraints imposed on diversification by the exclusion of individuals without mutualistic interactions from the mating pool would be lower for species with larger population sizes.

Second, increasing the number of different mutualistic partners constrained species-level diversification even when we relaxed our biological assumptions regarding how phenotype matching occurs, population densities, and the number of species under disruptive selection. The generality of these results suggests that stabilizing selection may often inhibit the diversification caused by disruptive selection in mutualisms involving many individual partners. Here, the sources of stabilizing selection are increased suboptimal matches arising from an increased number of different individual mutualistic partners. As the number of different interacting individuals increases, the stabilizing selection imposed by the escalating effects of trait mismatch on extreme phenotypes progressively counterbalances the disruptive selection caused by intraspecific competition. If rare extreme phenotypes need many different mutualistic partners, they face a higher degree of interaction mismatches than do common intermediate phenotypes. Furthermore, the stabilizing effect is amplified as more players become involved in mutualistic interactions and a larger proportion of the extreme phenotypes of the population are affected by suboptimal choices; the magnitude of such mismatches also increases (fig. 3 ).

The effects of intraspecific competition favoring extreme phenotypes may thus be reduced or even suppressed when mutualistic interactions involving multiple players are an important factor affecting fitness. Our simulations also show that increasing values of $\omega_{B}$, the number of different mutualistic partners with which the individuals of species $B$ interact, constrain the overall phenotypic variance within a clade, leading to progressively lower phenotypic distances among the emerging species. Decreased phenotypic distances between similar, related species may lead to strong interspecific competition, constraining intrapopulation
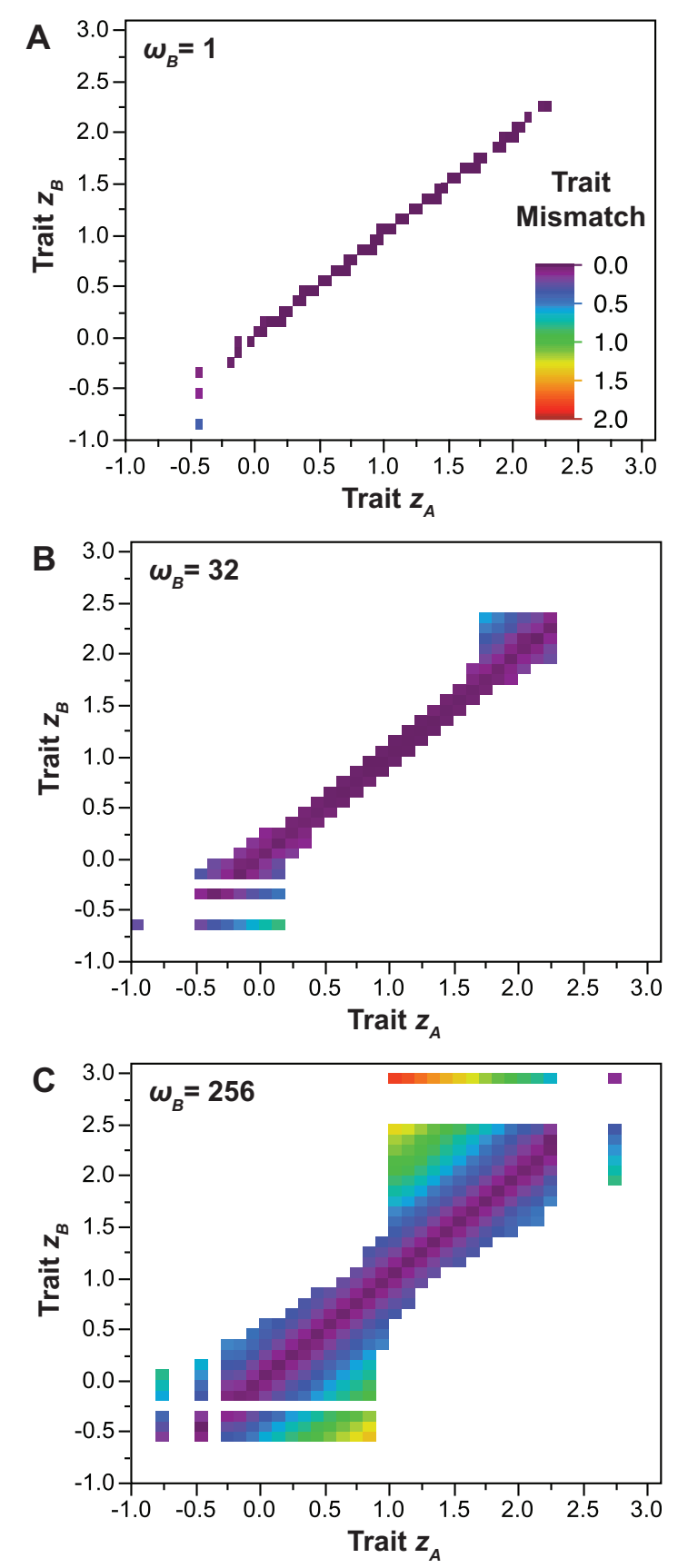

Figure 3: Phenotype mismatches in mutualistic interactions in which individuals of species $B$ have different numbers of mutualistic partners $\left(\omega_{B}\right)$. Terms $z_{A}$ and $z_{\mathrm{B}}$ are the values for the traits mediating the mutualism in species $A$ and $B$, respectively. Panels show the distribution of trait mismatches for all interaction events within a time step $t$, when individuals of species $B$ have a low number of different mutualistic partners $(A)$, intermediate number of different mutualistic partners $(B)$, or a high number of different mutualistic partners (C). The color gradient from purple (0) to red (2.00) represents increasing trait mismatch in interaction events. In all panels, $t=1$, $\lambda=1$, and $N_{A}=N_{B}=600$. 
variation. Thus, we hypothesize that selective pressures imposed by low-intimacy mutualisms add strength to the constraints imposed by interspecific competition to intrapopulation variation (Bolnick et al. 2003), potentially affecting ecological and evolutionary dynamics. For example, decreased phenotypic distances among the emerging species would impose sufficient interspecific competition to constrain species' niche width and avoid the continuous formation of extreme phenotypic clusters.

Our second simulation experiment suggests that adaptive diversification would emerge in mutualisms in which the degree of trait matching strongly affect fitness (high $\lambda$ ) and each individual interacts with few partners (low $\omega_{B}$ ) — even if only one species experiences the disruptive selection regime. Conversely, the simulations predict that adaptive diversification in low-intimacy mutualisms (large $\omega_{B}$ ) will often be constrained by strong stabilizing selection generated by phenotype mismatch in extreme phenotypes, counteracting the effect of disruptive selection. However, low-intimacy mutualistic assemblages hold up to 1 order of magnitude more species than high-intimacy assemblages in nature (fig. 4 and appendix). The departure between the patterns observed in nature and the predictions derived from our model provide insights on the role of different evolutionary processes in shaping the diversity of mutualistic assemblages.

Our model predicts that the species-rich assemblages formed by low-intimacy mutualisms are not likely to be a result of adaptive diversification promoted by intraspecific competition. As a consequence, other evolutionary processes are needed to explain the observed patterns of species richness in these systems. One possibility is that in low-intimacy mutualisms, which often form speciesrich networks, a highly interactive set of generalist species drives the evolution of the whole assemblages (Bascompte et al. 2003; Guimarães et al. 2011) and favor, through convergence and complementarity of traits, the incorporation of new nonrelated species into the assemblages (Thompson 2009). Therefore, low-intimacy mutualistic systems can become species-rich by aggregating nonrelated species over time (Ramírez et al. 2011). Another nonexclusive hypothesis is that some low-intimacy mutualisms, such as seed dispersal by vertebrates, may indirectly promote diversification by providing predictable resources and broadening geographic ranges. For example, species of mutualistic primates have large geographic ranges, which favor high geographic speciation rates and low extinction rates (Gómez and Verdú 2012).

On the other hand, our first simulation experiment does predict constraints to diversification in high-intimacy, obligate mutualisms (when individuals that fail to interact with mutualistic partners are excluded from the mating pool), such as pollination by flower parasites. Comple-

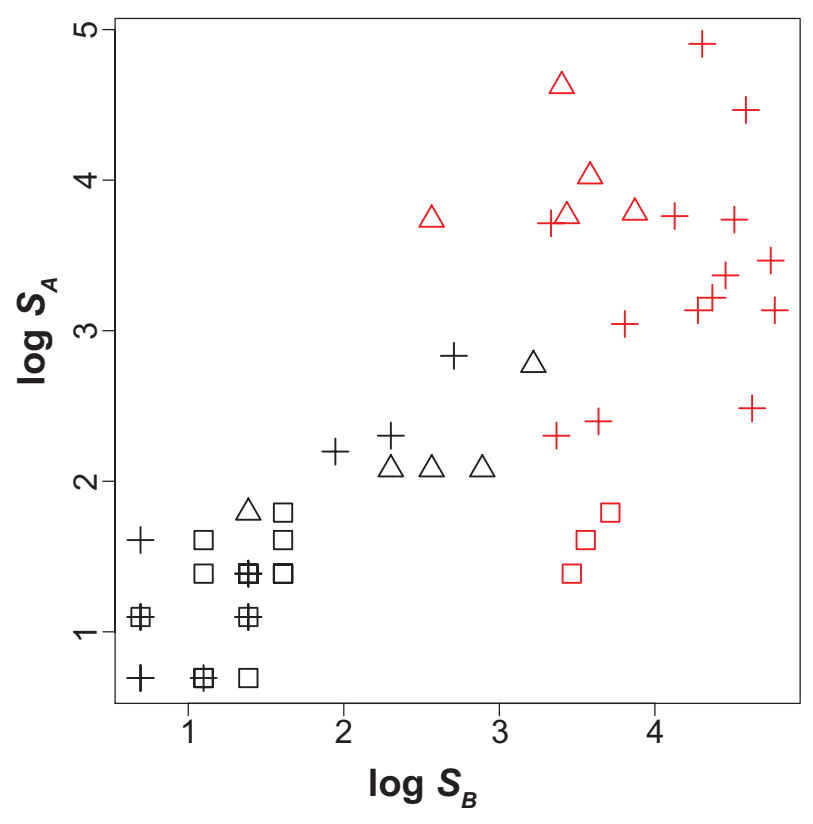

Figure 4: Species richness in natural mutualistic assemblages. Terms $S_{A}$ and $S_{B}$ indicate species richness for each group of mutualistic species (e.g., plants/pollinators). Black and red symbols indicate high- and low-intimacy mutualisms, respectively. Symbols represent different types of mutualism. Squares depict marine mutualisms (black = obligate interactions between anemonefish and their host species of sea anemones; red = cleaning mutualisms between cleaner species of shrimps and fishes and their fish clients). Triangles indicate ant-plant mutualisms (black = specialized protection mutualisms between ants and myrmecophyte plants; red = generalized ant-plant mutualisms, including protection mutualisms and ant-mediated seed dispersal). Crosses show pollination mutualisms (black = highly specialized, obligate interactions between plants and pollinating seed parasites; red = interactions between animal pollinators and flowering plants).

mentarily, other processes can also constrain species diversification in these high-intimacy mutualisms, leading to relatively species-poor systems. First, high-intimacy mutualisms can be more prone to species extinctions and coextinctions (Dáttilo 2012), which can contribute to lower local species richnesses despite a higher diversification potential. Additionally, high-intimacy mutualisms are embedded in tight coevolutionary processes involving complex sets of integrated traits (Thompson et al. 2013). As a consequence, high-intimacy systems are taxonomically constrained and have a very low potential to pull in nonrelated species into the interaction network. Indeed, events of addition of outside taxa to high-intimacy assemblages are few, even over tens of millions of years (Pellmyr 2003; Kawakita and Kato 2009).

The generality of our predictions rely on the major assumption that magic traits are involved in mutualistic interactions. An in-depth examination of this assumption 
requires more data on phenotypic selection and mating preferences in mutualisms. However, there is empirical evidence suggesting that magic traits can be found in intimate interactions, such as size-based assortative mating in anemonefish subjected to intense intraspecific competition (Hattori 2012). Moreover, the costs of assortative mating often constrain sympatric speciation (Bolnick and Fitzpatrick 2007). These costs may be reduced in highly intimate mutualisms as female choosiness becomes more viable due to a reduction of risks (e.g., predation) and search time. Moreover, the greater morphological, physiological, and behavioral integration between mutualistic partners found under intimate specialized mutualisms may favor the occurrence of automatic magic traits-the case in which prezygotic reproductive isolation by assortative mating is a by-product of trait evolution under disruptive selection (Servedio et al. 2011). Indeed, assortative mating is likely to occur when individuals mate in time- or spacedefined groups. For example, divergence in the timing of oviposition in relation to host phenology may initiate speciation in pollinating seed-parasitic globeflower flies (Ferdy et al. 2002) and the cospeciation of fig wasps and their hosts (Kiester et al. 1984). Conversely, the strength of assortative mating may be constrained in low-intimacy mutualisms due to viability costs (Rymer et al. 2010) Weak assortative mating may thus be an additional limiting factor constraining adaptive diversification in low-intimacy systems.

In conclusion, we argue here that conflicting selection pressures generated by intra- and interspecific ecological interactions have important consequences for our understanding of adaptive diversification. We have shown a general effect in which stabilizing selection generated by mutualistic trait complementarity constrains the adaptive diversification caused by intraspecific competition. These results hold under various biological assumptions and represent a first step in the study of conflicting selection during diversification in mutualistic assemblages. However, although trait complementarity is common (Dalsgaard et al. 2008), other interaction mechanisms, such as trait differences (Yoder and Nuismer 2010) or the interaction mechanisms assumed in game theoretical models of mutualisms (e.g., Gokhale and Traulsen 2012) are also recurrent in mutualisms and may impose alternative selection regimes that will interact with the disruptive selection caused by intraspecific competition to produce distinct outcomes in terms of species diversity. Investigating the effects of such alternative interaction mechanisms on adaptive diversification is a promising line of research addressing the broader challenge of describing the fundamental role of competition in the evolution of mutualisms (Jones et al. 2012).

\section{Acknowledgments}

We are indebted to D. Bolnick, P. Jordano, I. Karonen, G. Machado, F. Marquitti, M. Pires, T. Priklopil, E. SebastiánGonzáles, J. N. Thompson, R. Travitzki, G. S. van Doorn, and two anonymous reviewers for comments and suggestions. R.L.G.R. was supported by the São Paulo Research Foundation, Fundação de Amparo à Pesquisa do Estado de São Paulo (FAPESP; grant 2011/13054-6), and Coordenadoria de Aperfeiçoamento de Pessoal de Nível Superior (CAPES). P.R.G. was supported by FAPESP (grant 2009/54422-8). R.L.G.R. thanks the European Sciences Foundation program Frontiers in Speciation Research and the Finnish Doctoral Programme in Computational Sciences for the opportunity to attend the Helsinki Summer School on Mathematical Ecology and Evolution. The University of Nebraska-Lincoln supported J.P.G. through an Othmer Fellowship and the School of Biological Sciences Special Funds. D.H.H. was supported by the Department of Environmental Science, Policy, and Management at the University of California, Berkeley, and a postdoctoral fellowship from the Japan Society for the Promotion of Science.

\section{Literature Cited}

Anderson, B., and S. D. Johnson. 2008. The geographical mosaic of coevolution in a plant-pollinator mutualism. Evolution 62:220225.

Anderson, B., J. S. Terblanche, and A. G. Ellis. 2010. Predictable patterns of trait mismatches between interacting plants and insects. BMC Evolutionary Biology 10:204.

Bascompte, J., and P. Jordano. 2007. Plant-animal mutualistic networks: the architecture of biodiversity. Annual Review of Ecology, Evolution and Systematics 38:567-593.

Bascompte, J., P. Jordano, C. J. Melián, and J. Olesen. 2003. The nested assembly of plant-animal mutualistic networks. Proceedings of the National Academy of Sciences of the USA 100:9383-9387. Bentley, B. L. 1977. Extrafloral nectaries and protection by nectaries and pugnacious body guards. Annual Review of Ecology and Systematics 8:407-427.

Bhattacharyay, A., and B. Drossel. 2005. Modeling coevolution and sympatric speciation of flowers and pollinators. Physica A 345: 159-172.

Bolnick, D. I., and B. M. Fitzpatrick. 2007. Sympatric speciation: models and empirical evidence. Annual Review of Ecology, Evolution, and Systematics 38:459-487.

Bolnick, D. I., R. Svanbaeck, J. A. Fordyce, L. H. Yang, J. M. Davis, C. D. Hulsey, and M. L. Forister. 2003. The ecology of individuals: incidence and implications of individual specialization. American Naturalist 161:1-28.

Bond, W. J. 1994. Do mutualisms matter? assessing the impact of pollinator and disperser disruption on plant extinction. Philosophical Transactions of the Roval Society B: Biological Sciences 344:83-90.

Bronstein, J. L. 2009. Mutualism and symbiosis. Pages 233-238 in 
S. A. Levin, ed. The Princeton guide to ecology. Princeton University Press, Princeton, NJ.

Bronstein, J. L., R. Alarcón, and M. Geber. 2006. The evolution of plant-insect mutualisms. New Phytologist 172:412-428.

Bshary, R., A. Hohner, K. Ait-el-Djoudi, and H. Fricke. 2006. Interspecific communicative and coordinated hunting between groupers and giant moray eels in the Red Sea. PLoS Biology 4:e431.

Bürger, R. 2002. On a genetic model of intraspecific competition and stabilizing selection. American Naturalist 160:661-682.

Bürger, R., K. A. Schneider, and M. Willensdorfer. 2006. The conditions for speciation through intraspecific competition. Evolution 60:2185-2206.

Dalsgaard, B., A. M. Martin-Gonzalez, J. M. Olesen, A. Timmermann, L. H. Andersen, and J. Ollerton. 2008. Pollination networks and functional specialization: a test using Lesser Antillean plant-hummingbird assemblages. Oikos 117:789-793.

Dáttilo, W. 2012. Different tolerances of symbiotic and nonsymbiotic ant-plant networks to species extinctions. Network Biology 2:127138.

Dieckmann, U., M. Doebeli, J. A. J. Metz, and D. Tautz. 2004. Adaptive speciation. Cambridge University Press, Cambridge.

Doebeli, M. 2011. Adaptive diversification. Princeton University Press, Princeton, NJ.

Doebeli, M., and U. Dieckmann. 2000. Evolutionary branching and sympatric speciation caused by different types of ecological interactions. American Naturalist 156(suppl.):S77-S101.

Felsenstein, J. 1979. Excursions along the interface between disruptive and stabilizing selection. Genetics 93:773-795.

Ferdy, J.-B., L. Després, and B. Godelle. 2002. Evolution of mutualism between globeflowers and their pollinating flies. Journal of Theoretical Biology 217:219-234.

Fonseca, C. R. 1999. Amazonian ant-plant interactions and the nesting space limitation hypothesis. Iournal of Tropical Ecology 15: $807-825$.

Fonseca, C. R., and G. Ganade. 1996. Asymmetries, compartments and null interactions in an Amazonian ant-plant community. Journal of Animal Ecology 65:339-347.

Fontaine, C., P. R. Guimarães Jr., S. Kéfi, N. Loeuille, J. J. Memmott, W. H. van der Putten, F. J. F. van Veen, and E. Thébault. 2011. The ecological and evolutionary implications of merging different types of networks. Ecology Letters 14:1170-1181.

Gokhale, C. S., and A. Traulsen. 2012. Mutualism and evolutionary multiplayer games: revisiting the Red King. Proceedings of the Royal Society B: Biological Sciences 279:4611-4616.

Gómez, J. M., and F. Perfectti. 2012. Fitness consequences of centrality in mutualistic individual-based networks. Proceedings of the Roval Societv B: Biological Sciences 279:1754-1760.

Gómez, J. M., and M. Verdú. 2012. Mutualism with plants drives primate diversification. Systematic Biology 61:567-577.

Guerrero, A. M., and A. Tye. 2009. Darwin's finches as seed predators and dispersers. Wilson Journal of Ornithology 121:752-764.

Guimarães, P. R., Jr., P. Jordano, and J. N. Thompson. 2011. Evolution and coevolution in mutualistic networks. Ecology Letters 14:877885.

Guimarães, P. R., Jr., V. Rico-Gray, P. S. Oliveira, T. J. Izzo, S. F. Reis, and J. N. Thompson. 2007. Interaction intimacy affects structure and coevolutionary dynamics in mutualistic networks. Current Biology 17:1797-1803.

Hattori, A. 2012. Determinants of body size composition in limited shelter space: why are anemonefishes protandrous? Behavioral Ecology 23:512-520.

Herrera, C. M. 1995. Plant-vertebrate seed dispersal systems in the Mediterranean: ecological, evolutionary, and historical determinants. Annual Review of Ecology and Systematics 26:705-727.

Jones, E. I. 2010. Optimal foraging when predation risk increases with patch resources: an analysis of pollinators and ambush predators. Oikos 119:835-840.

Jones, E. I., J. L. Bronstein, and R. G. Ferrière. 2012. The fundamental role of competition in the ecology and evolution of mutualisms. Annals of the New York Academy of Sciences 1256:66-88.

Kawakita, A., and M. Kato. 2009. Repeated independent evolution of obligate pollination mutualism in the Phyllantheae-Epicephala association. Proceedings of the Roval Society B: Biological Sciences 276:417-426.

Kiester, A. R., R. Lande, and D. W. Schemske. 1984. Models of coevolution and speciation in plants and their pollinators. American Naturalist 124:220-243.

Kingsolver, J. G., H. E. Hoekstra, J. M. Hoekstra, D. Berrigan, S. N. Vignieri, C. E. Hill, A. Hoang, P. Gibert, and P. Beerli. 2001. The strength of phenotypic selection in natural populations. American Naturalist 157:245-261.

Lankinen, Å., and M. C. Larsson. 2009. Conflicting selection pressures on reproductive functions and speciation in plants. Evolutionary Ecology 23:147-157.

Lanza, J., G. C. Smith, S. Sack, and A. Cash. 1995. Variation in nectar volume and composition of Impatiens capensis at the individual, plant, and population levels. Oecologia (Berlin) 102:113-119.

Léotard, G., A. Saltmarsh, F. Kjellberg, and D. McKey. 2008. Mutualism, hybrid inviability and speciation in a tropical ant-plant. Journal of Evolutionary Biology 21:1133-1143.

Minta, S., K. Minta, and D. Lott. 1992. Hunting associations between badgers (Taxidea taxus) and coyotes (Canis latrans). Journal of Mammalogy 73:814-820.

Ollerton, J. 2006. "Biological barter": patterns of specialization compared across different mutualisms. Pages 411-435 in N. M. Waser and J. Ollerton, eds. Plant-pollinator interactions: from specialization to generalization. University of Chicago Press, Chicago.

Palmer, T. M., M. L. Stanton, and T. P. Young. 2003. Competition and coexistence: exploring mechanisms that restrict and maintain diversity within mutualist guilds. American Naturalist 162(suppl.): S63-S79.

Pellmyr, O. 2003. Yuccas, yucca moths, and coevolution: a review. Annals of the Missouri Botanical Gardens 90:35-55.

Pires, M. M., and P. R. Guimarães Jr. 2013. Interaction intimacy organizes networks of antagonistic interactions in different ways. Journal of the Royal Society Interface. doi:10.1098/rsif.2012.0649.

Ramírez, S. R., T. Eltz, M. K. Fujiwara, G. Gerlach, B. GoldmanHuertas, N. D. Tsutsui, and N. E. Pierce. 2011. Asynchronous divergence in a specialized plant-pollinator mutualism. Science 333:1742-1746.

Ricciardi, F., Massimo B., and J. Ollerton. 2010. Assemblage and interaction structure of the anemonefish-anemone mutualism across the Manado Region of Sulawesi, Indonesia. Environmental Biology of Fishes 87:333-347.

Rosenzweig, M. L. 1978. Competitive speciation. Biological Journal of the Linnean Society 10:275-289.

Rymer, P. D., S. D. Johnson, and V. Savolainen. 2010. Pollinator behaviour and plant speciation: can assortative mating and dis- 
ruptive selection maintain distinct floral morphs in sympatry? New Phytologist 188:426-436.

Servedio, M. R., G. S. van Doorn, and M. Kopp. 2011. Magic traits in speciation: "magic" but not rare? Trends in Ecology and Evolution 26:389-397.

Siepielski, A. M., and C. W. Benkman. 2009. Conflicting selection from an antagonist and a mutualist enhances phenotypic variation in a plant. Evolution 64:1120-1128.

Stevens, M. H. H., M. Sanchez, J. Lee, and S. E. Finkel. 2007. Diversification rates increase with population size and resource concentration in an unstructured habitat. Genetics 177:2243-2250.

Strauss, S. Y., and R. E. Irwin. 2004. Ecological and evolutionary consequences of multispecies plant-animal interactions. Annual Review of Ecology, Evolution and Systematics 35:435-466.

Tarayre, M., G. Bowman, A. Schermann-Legionnet, M. Barat, and A. Atlan. 2007. Flowering phenology of Ulex europaeus: ecological consequences of variation within and among populations. Evolutionary Ecology 21:395-409.

Thompson, J. N. 2005. The geographic mosaic of coevolution. University of Chicago Press, Chicago.
2009. Coevolution. Pages 247-252 in S. A. Levin, ed. The Princeton guide to ecology. Princeton University Press, Princeton, NJ.

Thompson, J. N., C. Schwind, P. R. Guimarães Jr., and M. Friberg. 2013. Diversification through multitrait evolution in a coevolving interaction. Proceedings of the National Academy of Sciences of the USA 110:11487-11492.

Waser, N. M., L. Chittka, M. V. Price, N. M. Williams, and J. Ollerton. 1996. Generalization in pollination systems, and why it matters. Ecology 77:1043-1060.

Weiblen, G. D. 2002. How to be a fig wasp. Annual Review of Entomology 47:299-330.

Yoder, J. B., and S. L. Nuismer. 2010. When does coevolution promote diversification? American Naturalist 176:802-817.

Associate Editor: William F. Morris Editor: Judith L. Bronstein

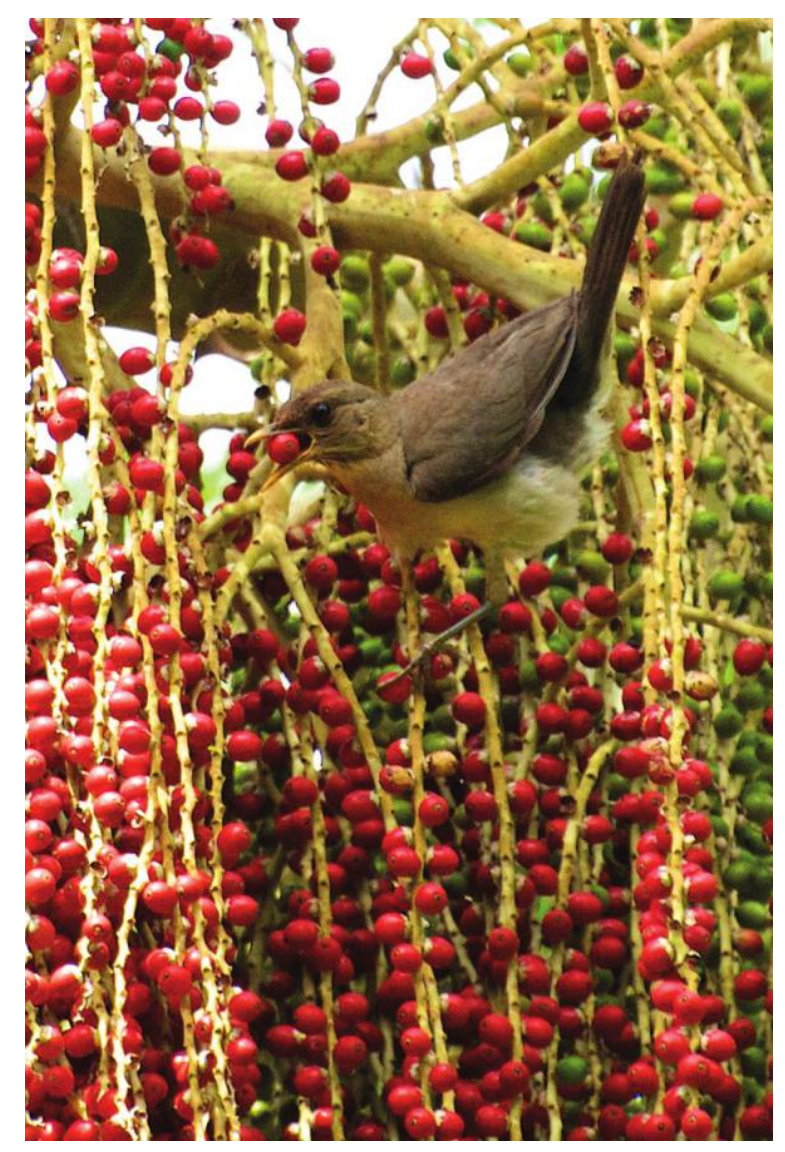

A creamy-bellied thrush (Turdus amaurochalinus) feeding on fruits of the bangalow palm (Archontophoenix cunninghamiana) in the Atlantic Forest of Brazil. Photo credit: Pedro Jordano. 


\section{ONLINE APPENDIX A - SUPPORTING INFORMATION}

\section{Conflicting selection in the course of adaptive diversification: the interplay between mutualism and intraspecific competition}

Rafael L. G. Raimundo, Jean P. Gibert, David H. Hembry, and Paulo R. Guimarães Jr

\section{Contents}

Impact of the degree of trait matching on mating probabilities ..................................................... 2

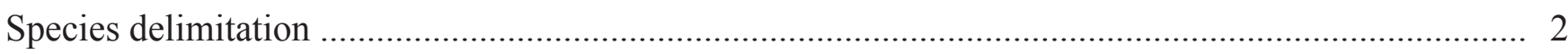

Asymptotic levels of diversity and stability of diversification patterns ............................................ 3

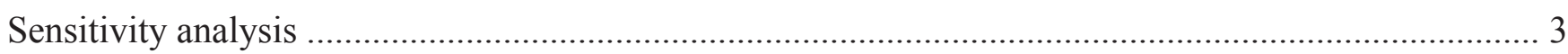

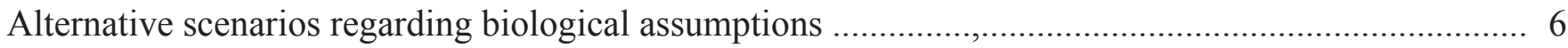

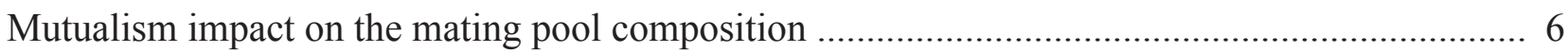

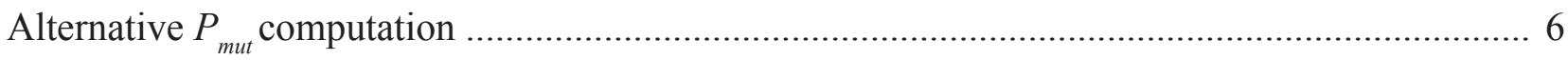

Symmetric constraint on the number of individual mutualistic partners .................................... 11

Relaxing the assumption of both species under disruptive selection........................................... 11

Relaxing the assumption of equal and constant population sizes ........................................... 12

Males under selective pressures arising from ecological interactions ....................................... 13

Species richness in natural mutualistic assemblages ................................................................. 13

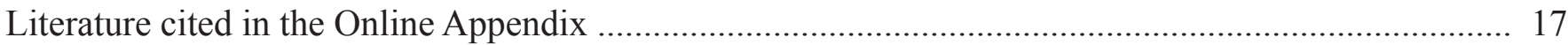




\section{Impact of the degree of trait matching on mating probabilities}

We used the parameter $\lambda$ (equation 4 , main text) to describe the effect of the degree of trait matching of mutualistic interactions on the reproductive output of individuals. Weassumed that $\lambda$ varies between 0 and 1 . If $\lambda=1$, increasing mismatches rapidly impose great reproductive loss (fig. A1-A). Mismatched interactions are progressively less penalized under decreasing values of $\lambda$ (figs. A1-B-C). As the parameter approaches 0 , the reproductive impact of trait matching becomes uniform within the population (fig. A1-D).

\section{Species delimitation}

We applied a phenotypic clustering criterion (PCC) to delimit species emerging in our simulations. Phenotypic clusters are often used for species delimitation in adaptive speciation models (Doebeli and Dieckmann 2000). We built an algorithm based on the discontinuous distribution of a trait $z$ to identify clusters defined by phenotypic discontinuities. The algorithm uses a parameter $l$ to define the degree of discontinuity separating ecologically differentiated and reproductively isolated individuals. To test the consistency of PCC in determining species identities in our simulations, we (i) compared species richness $(S)$ computed under different $l$ values to the number of lineages observed in phenotypic trajectory plots describing trait divergence through time, (ii) tracked individuals' genealogies to check for the existence of hybrids, i.e., individuals with parents belonging to different species, and (iii) examined phenotypic clusters to check if they hold individuals descending from more than one species.With regards to (i), species richness detected by PCC is overestimated if $l<0.1$ units of $z$, since individuals isolated by small phenotypic discontinuities, as those found in the extreme of each species distributions, are classified as unique species. For example, when $l=0.05$, the species delimitation rule recognizes up to 75 species deriving from species $A$ (fig. A2, blue line), whereas the actual number of lineages is much lower (fig. A3). A better estimate for species richness within our adaptive diversification model is
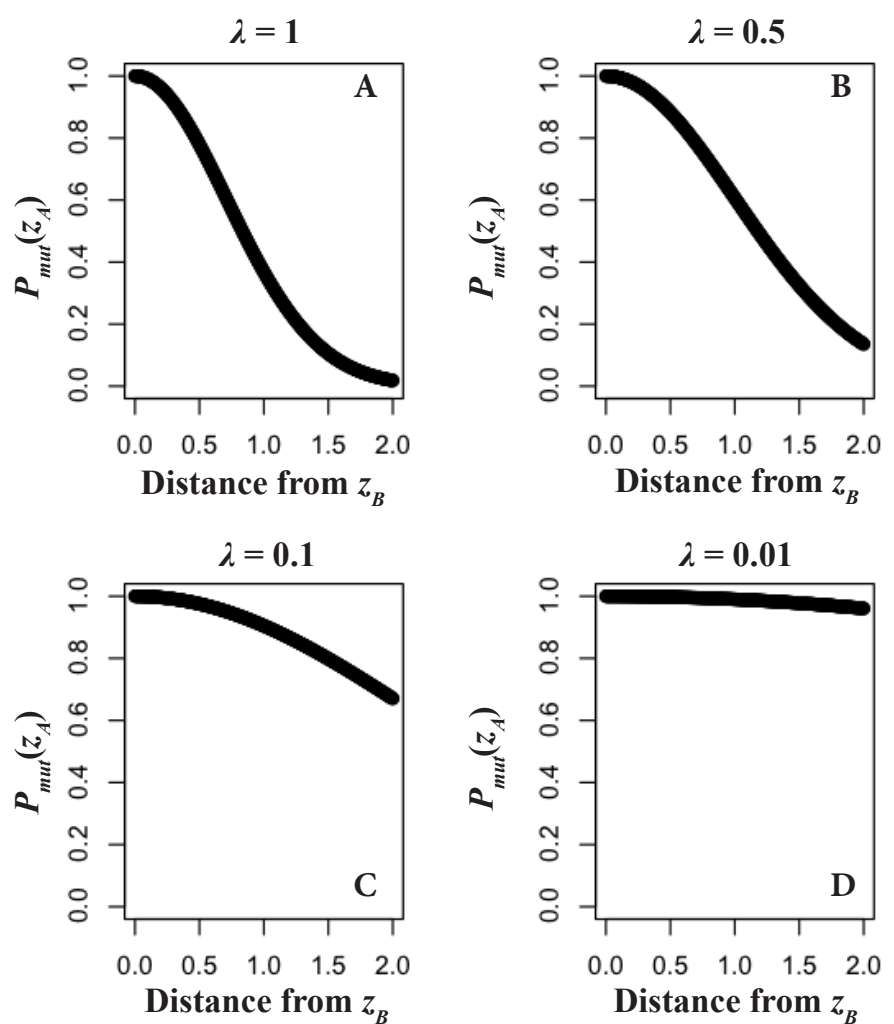

Figure A1. The reproductive outcome of mutualistic interactions for individuals of species $A, P_{\text {mut }}\left(z_{A}\right)$, considering a fixed value of $z_{B}=1$ and different values of $\lambda$, the parameter describing the effect of the trait matching of mutualistic interactions on individual mating probabilities (see equation 4 in the main text).

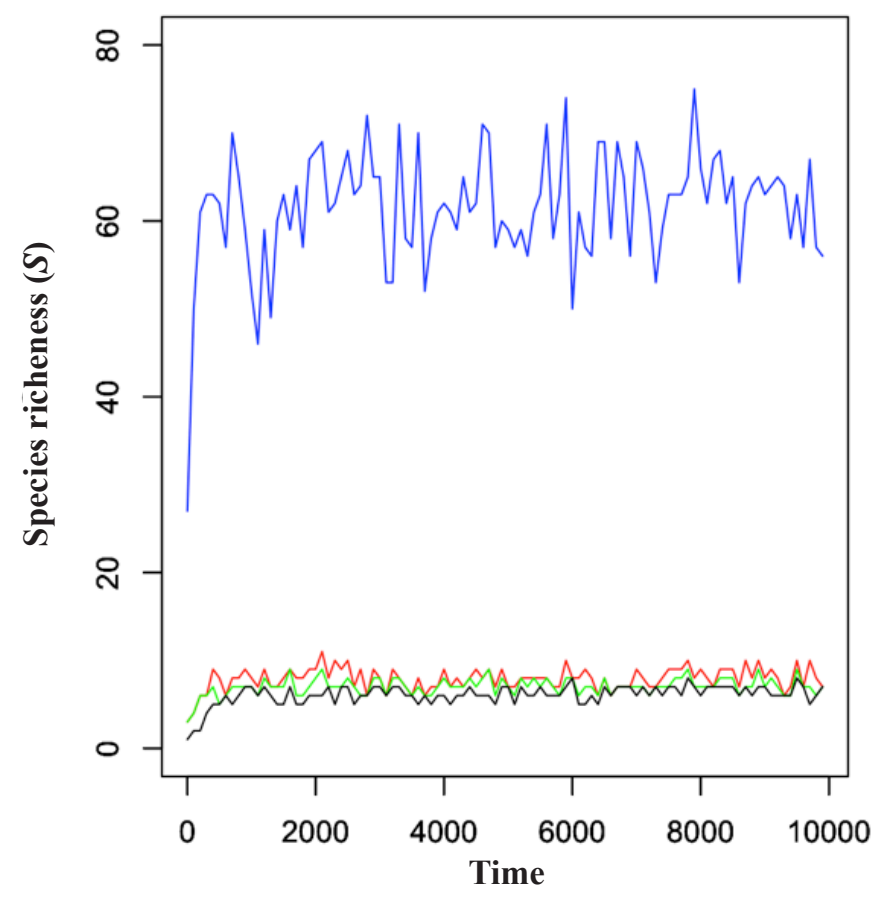

Figure A2. Temporal variation in species richness $(S)$ according to the limiting parameter, 1 , used in the phenotypic clustering criterion (PCC). $S$ is the sum of clades derived from species $A$ and $B$ emerging in simulations of adaptive diversification. Lines describe different values of $l$, which defines the phenotypic discontinuity determining species borders. Blue, $l=0.05$; red, $l=0.1$; green, $l=0.2$; black, $l=0.4$. Same parameters as those of Figure 1 (see main text) through 10,000 time steps. 
found when $0.1 \leq l \leq 0.2$. Values of $l>>0.2$ underestimate species richness since ecologically specialized and reproductively isolated clusters are merged within the same species (fig. A2, black line). When $l=0.1$, even incipient divergence between clusters are computed in the richness count (fig. A3-B), whereas $l$ $=0.2$ provides a more accurate approximation for the number of lineages observed in phenotypic trajectory plots (figs. A3-B, A3-D). Based on these results, we chose a value of $l=0.2$ for the species delimitation parameter used in our species richness analyses. With regards to (ii), phenotypic clusters emerging in our model can be interpreted as reproductively isolated units, since $100 \%$ of individuals had both parents belonging to the same cluster/species $(\mathrm{n}=$ 60,000 individuals sampled during 100 time steps uniformly distributed through 10,000 generations, $l=0.2$ ). Therefore, each phenotypic cluster results from assortative mating within a small number of genealogically linked individuals (families) (fig. A3A). Finally, with regards to (iii), only a small fraction of individuals within a given cluster eventually descend from multiple species. We found that 97.5 $\pm 10 \%$ of individuals per generation are assembled in clusters in which all organisms descend from a unique parental species $(\mathrm{n}=60,000$ sampled during 100 time steps uniformly distributed through 10,000 generations, $l=0.2$ ).

\section{Asymptotic levels of diversity and stability of diversification patterns}

We used the PCC algorithm to describe the temporal variation in species richness $(S)$ through 10,000 generations. The diversification patterns remained stable after 10,000 generations (Figure A4). Species richnesses reached asymptotic levels before 1,000 time steps (fig. A5). These results are consistent under different values of the mutualism attributes $\left(\omega_{B}\right.$ and $\lambda$ ). Similar asymptotic levels were also observed for another diversity measure, the Shannon-Weaver index $\left(H^{\prime}\right)$. We thus proceeded to compute species richness in simulation replicates using the number of species found by the PCC algorithm after 1,000 time steps.

\section{Sensitivity analysis}

We performed a sensitivity analysis using Latin hypercube sampling (Stein 1987, Carnell 2009) to explore the parametric space and identify parameter combinations resulting in greater species diversification. This sensitivity test considered a mutualism defined by $\omega_{B}=\lambda=1$ to explore the model dynamics under variable initial trait variances $\left(\sigma_{A}=\sigma_{B}\right)$, strengths of stabilizing selection $(\gamma)$ and strengths of intraspecific competition $(c)$. We ran 20 simulations encompassing the sampled parametric combinations (Table A1) under four population sizes $\left(N_{A}=N_{B}=75,150,300\right.$, and 600 individuals), thus totaling 80 simulations. Figure A6 (A-D) shows the results of the sensitivity analysis for $N_{A}=N_{B}=150,300$, and 600 individuals.

Varying population sizes allowed us to assess the extent to which asymptotic richness depends on the number of individuals in the population. Higher population sizes associated with strong intraspecific competition and low environmental stabilizing selection promote the greatest degrees of species diversification $\left(\sigma_{A}=\sigma_{B}=0.58, \gamma=0.001, c=0.74\right)$. This parameter combination defined the baseline scenario for two simulation experiments designed to explore the hypothesis that mutualism attributes constrain adaptive diversification (see the main text).

The highest diversification degree - a radiationlike pattern with recurrent extinctions - thus arises when the strength of stabilizing selection, $\gamma$, is low $(<0.1)$. This pattern is particularly evident in larger populations (figs. A6s1-s2). When $0.5>\gamma>0.1$, populations split into several stable phenotypic lineages (figs. A6s3-s10), except when the strength of intraspecific competition (c) decreases, which leads to a single branching event (fig, A6s8). When $\gamma>0.5$, results converge to a single branching event regardless of population size (fig. A6s11-s20), except if $c$ is low, in which case larger populations may hold polymorphisms without bifurcating (fig. A6s1718). When $\gamma>0.1$, most small populations $(75,150$ individuals) undergo a single branching event, or branching-extinction cycles if $c$ is near its maximum (fig. A6s6). 
Table A1. Parameter combinations used for sensitivity analysis.

\begin{tabular}{cccc}
\hline Sample & Initial trait variance $\left(\sigma_{A}=\sigma_{B}\right)$ & Strength of stabilizing selection $(\gamma)$ & Strength of intraspecific competition $(c)$ \\
\hline s1 & 0.58 & 0.00 & 0.74 \\
s2 & 0.76 & 0.08 & 0.56 \\
s3 & 0.61 & 0.10 & 0.62 \\
s4 & 0.73 & 0.15 & 0.54 \\
s5 & 0.05 & 0.24 & 0.39 \\
s6 & 0.19 & 0.28 & 0.97 \\
s7 & 0.01 & 0.31 & 0.49 \\
s8 & 0.32 & 0.36 & 0.21 \\
s9 & 0.52 & 0.40 & 0.79 \\
s10 & 0.30 & 0.46 & 0.88 \\
s11 & 0.92 & 0.55 & 0.07 \\
s12 & 0.25 & 0.56 & 0.02 \\
s13 & 0.66 & 0.62 & 0.16 \\
s14 & 0.41 & 0.69 & 0.11 \\
s15 & 0.48 & 0.74 & 0.30 \\
s16 & 0.87 & 0.79 & 0.26 \\
s17 & 0.38 & 0.81 & 0.80 \\
s18 & 0.14 & 0.88 & 0.94 \\
s19 & 0.81 & 0.91 & 0.44 \\
s20 & 0.97 & 0.98 & 0.70 \\
\hline & & &
\end{tabular}

A
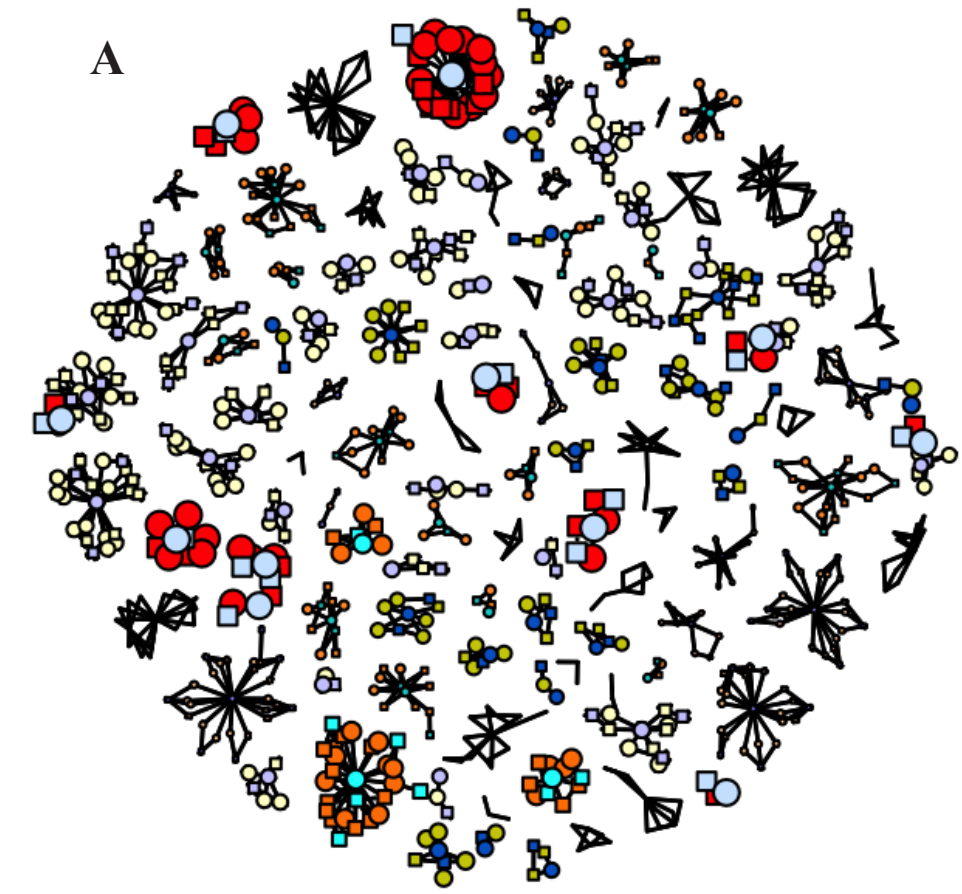

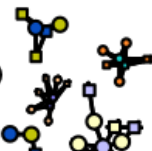<smiles>[Y]</smiles>

Figure A3. Species delimitation according to the phenotypic clustering criterion (PCC). (A) Reproductive networks of parents (cold colors) and offspring (hot colors) for the last $(1,000$ th) generation with $l=0.2$. Each color represents a unique species derived from species $A$. Squares show males and circles indicate females. Each phenotypic cluster in the offspring if formed by sets of families descending from the same parental species. (B) Distribution of offspring individuals in clusters, here interpreted as species, for $l=0.1$ ( 8 species) and $l=0.2$ (7 species). Species' colors are the same used in (A). (C) Frequency of individuals within each cluster for the $1,000^{\text {th }}$ generation and (D) Phenotypic trajectories for the trait $z$ through 1,000 generations. The last generation in the plot correspond to the data used in (A), (B), and (C).
B $\begin{aligned} & l=0.1 \\ & l=0.2\end{aligned} \infty \infty$
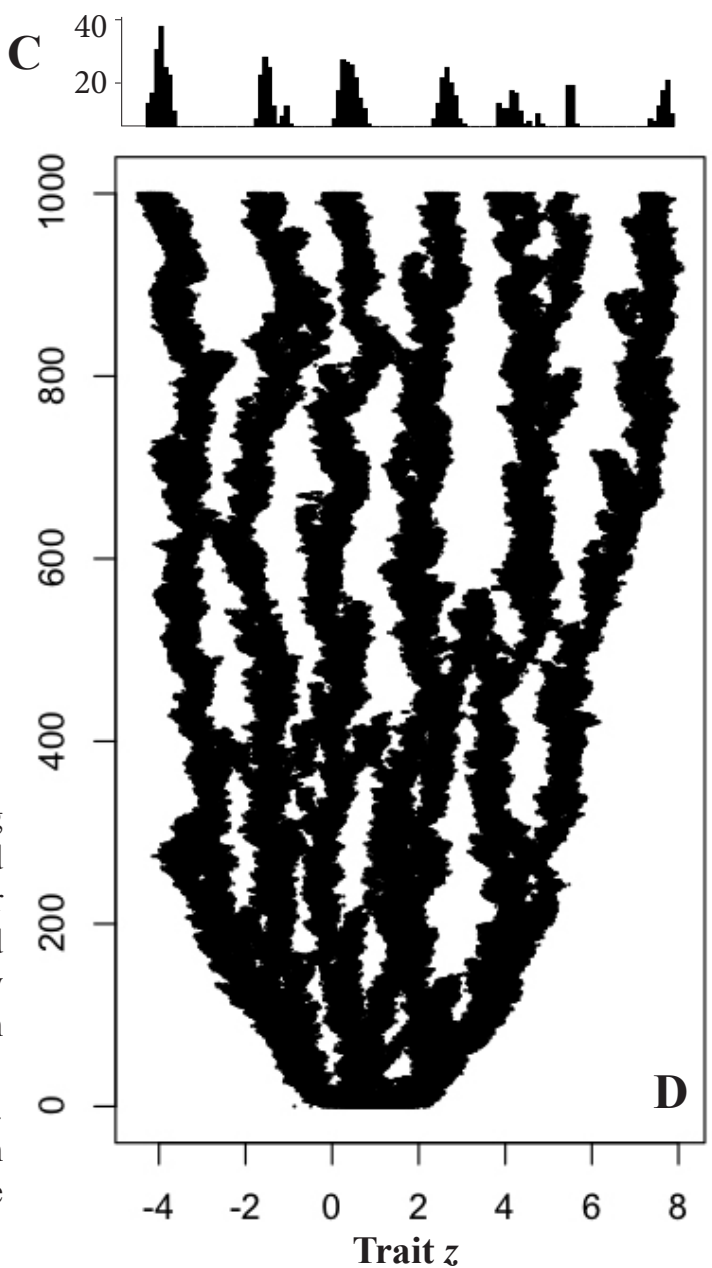


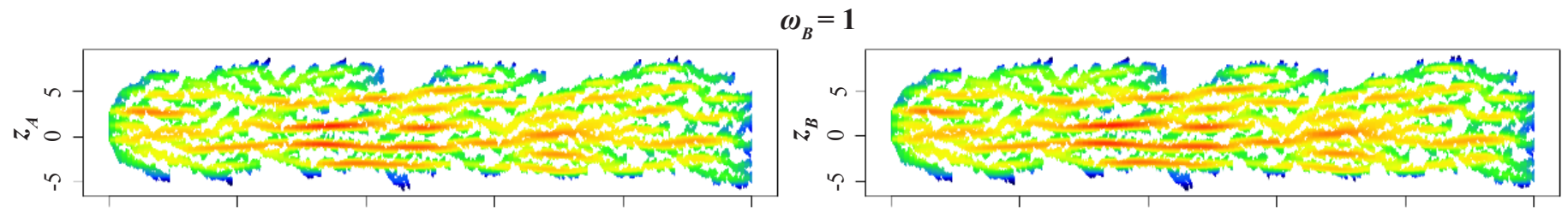

$\omega_{B}=4$
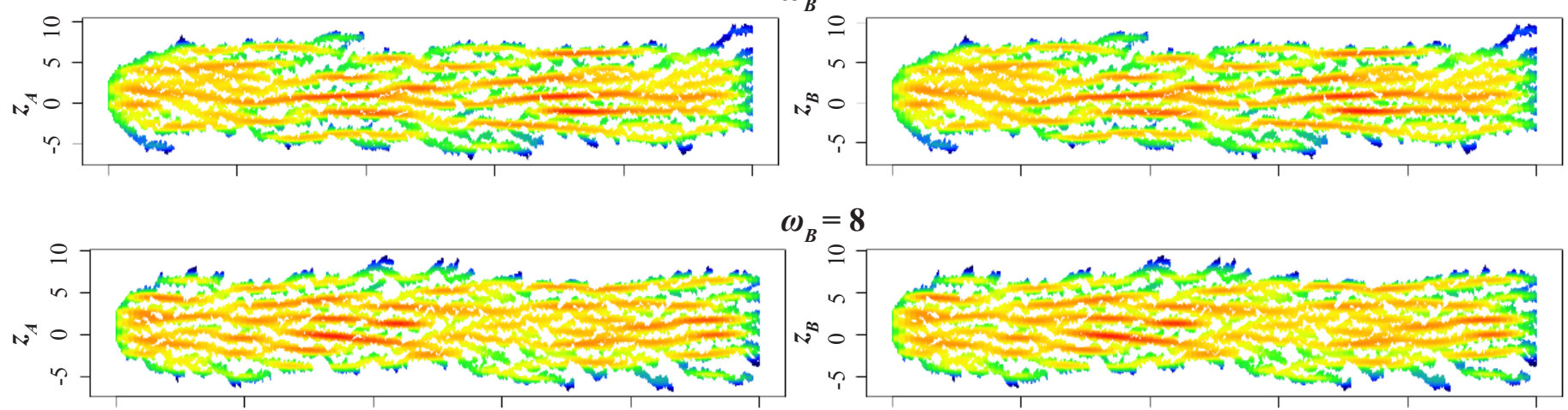

$\omega_{B}=16$

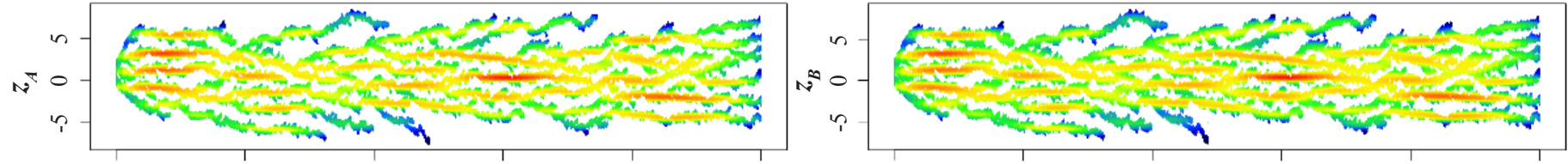

$\omega_{B}=32$

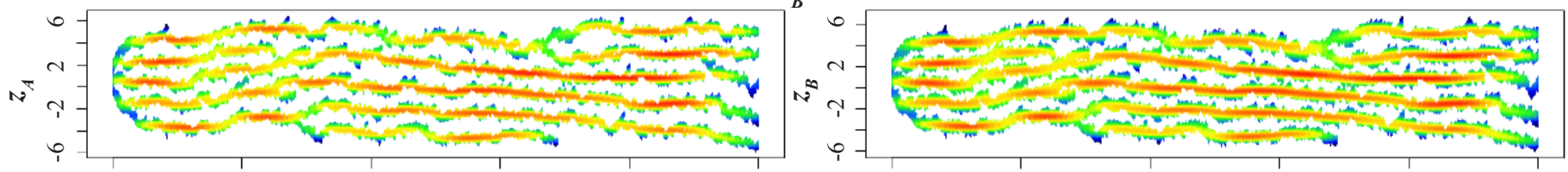

$\omega_{B}=64$

$N_{0}^{T}$

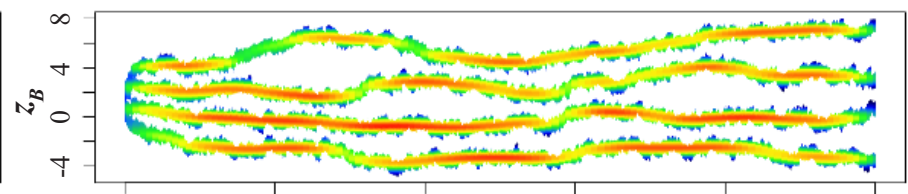

$\omega_{B}=128$

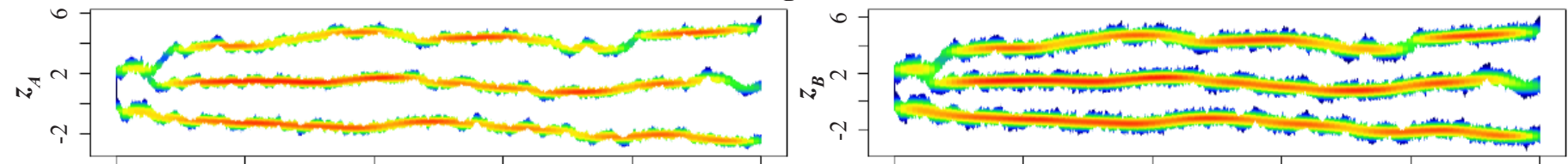

$\omega_{B}=256$
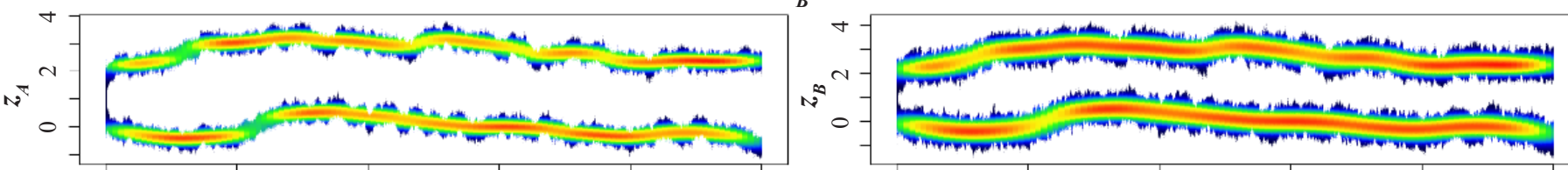

$\omega_{B}=\mathbf{5 1 2}$
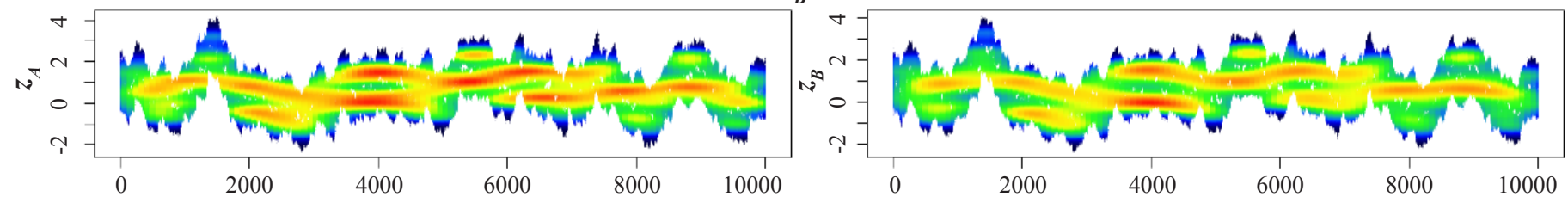

Time

Figure A4. Phenotypic trajectories under diverse mutualism attributes through 10,000 time steps. The values at the left of each panel indicate the number of mutualistic partners with which each individual of species $B$ interacted $\left(\omega_{B}\right)$. Other parameters are the same as in Figure 1 (see the main text). Temperature colors depict density of individuals, ranging from high (hot colors) to low (cold colors). 

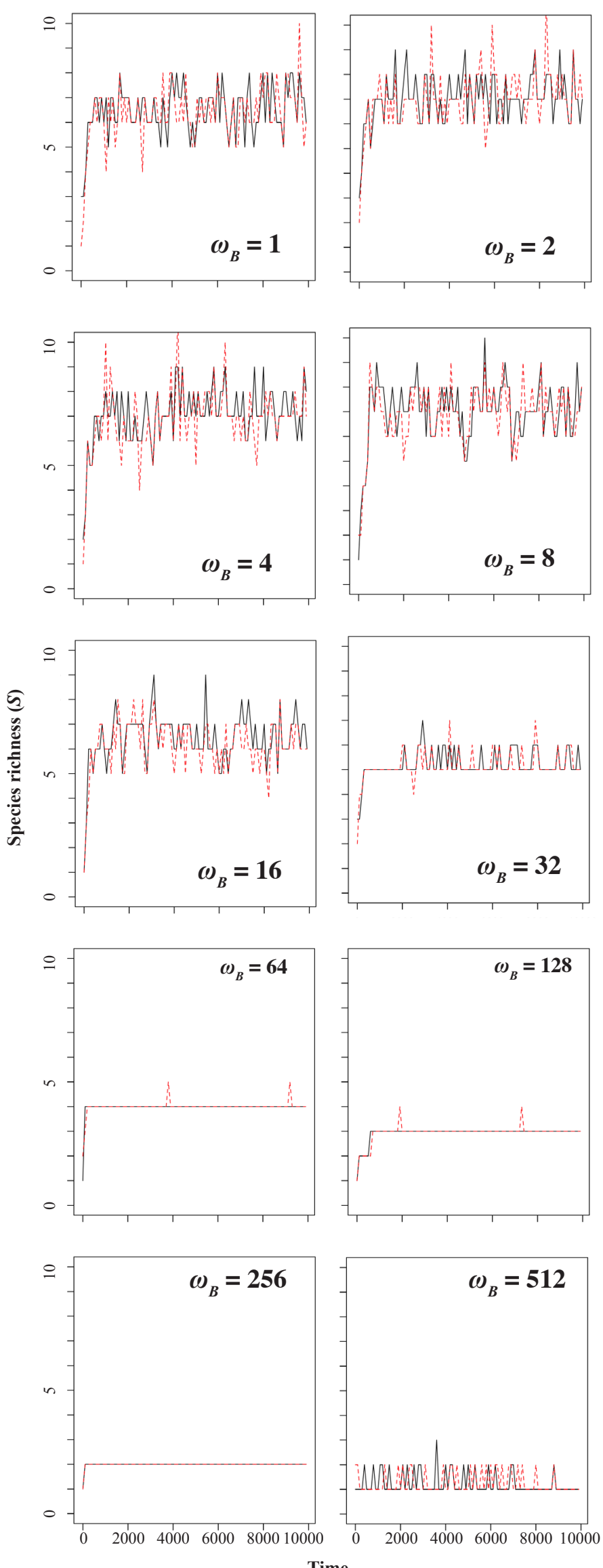

Time

Figure A5. Temporal variation in number of species $(S)$ derived from species $A$ (black lines) and from species $B$ (red lines) under varying $\omega_{B}$. Numbers inside each panel indicate the number $\omega_{B}$ of mutualistic partners with which each species $B$ individual interacted. Other parameters are the same as in Figure 1 (see the main text).

\section{Alternative scenarios regarding biological assumptions}

In the following sections, we explore the model dynamics under alternative biological assumptions in relation to those of our baseline model.

\section{Mutualism impact on the mating pool composition}

In our model, species $A$ is not limited regarding the number of interspecific partners individuals may have. Therefore, some individuals may interact much more times than others, whereas a proportion of the population may remain without mutualistic partners (fig. A7). The reproductive consequences for individuals remaining without any mutualistic partners could be: (i) reduced mating probabilities or (ii) preclusion from the mating pool. Indeed, highly intimate mutualisms can potentially filter individuals from the mating pool.

We thus ran additional simulations in which we assumed that if individuals of species $A$ were not selected by any mutualistic partner, they were also unable to mate $\left(P_{\text {mat }}=0\right)$. Adaptive diversification is constrained when highly intimate mutualisms $\left(\omega_{B}\right.$ $=\lambda=1$ ) act as mating filters (see the main text for detailed results). The relative frequency of extreme phenotypes with relatively high mating probabilities decreases, since those individuals that suffered low intraspecific competition but did not interact are precluded from the mating pool.

\section{Alternative $\mathrm{P}_{\text {mut }}$ computation}

The fitness component $P_{m u t}$, describing the gain obtained by individuals from mutualistic interactions, was computed by summing up the outcome of each interaction event. Although $P_{m u t}$ is a standardized fitness component (equation 4 in main text), we tested if the progressive limit imposed to diversification by increasing values of $\omega_{B}$ could simply be a consequence of an additive effect of interaction events. If $P_{m u t}$ is computed based on the mean benefit acquired by individuals, and is therefore proportional to mean trait complementarity, the effect of $\omega_{B}$ on adaptive diversification is consistent with the results of our simulation experiment (fig. A8). 


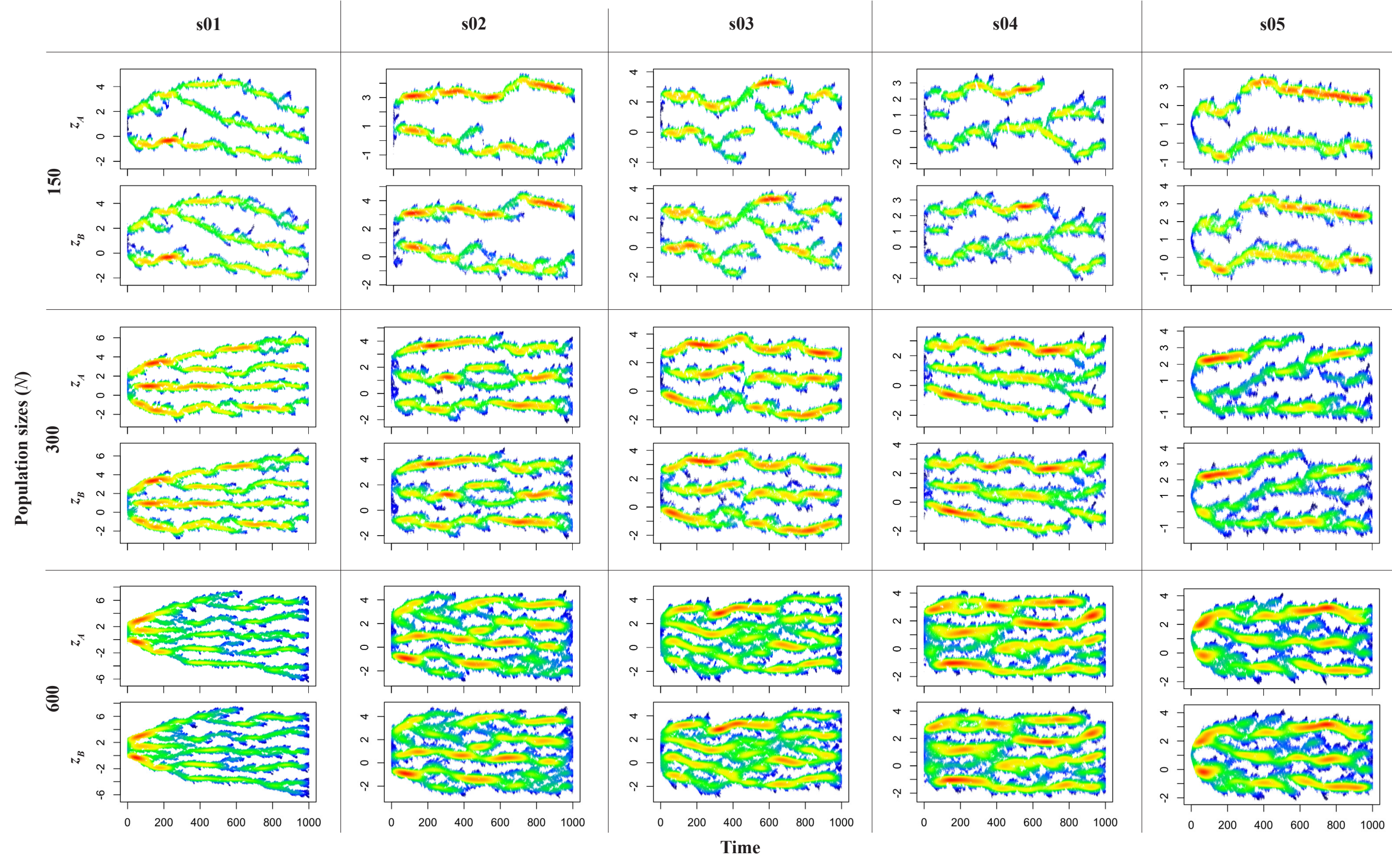

Figure A6-A. Phenotypic trajectories plots for traits $z_{A}$ and $z_{B}$ under parameter combinations (s01-s05) obtained using Latin Hypercube sampling. See Table 1 for descriptions of the parameters and their values. The columns present phenotypic trajectories under each parameter combination and rows show results for three population sizes $\left(N_{A}=N_{B}=150,300,600\right)$. Abscissae depict the number of time steps for which each simulation ran and follow the labels in the last row, ranging from 0 to 1000 . Colors represent density of individuals throughout the phenotypic space. $\omega_{B}=\lambda=1$. 


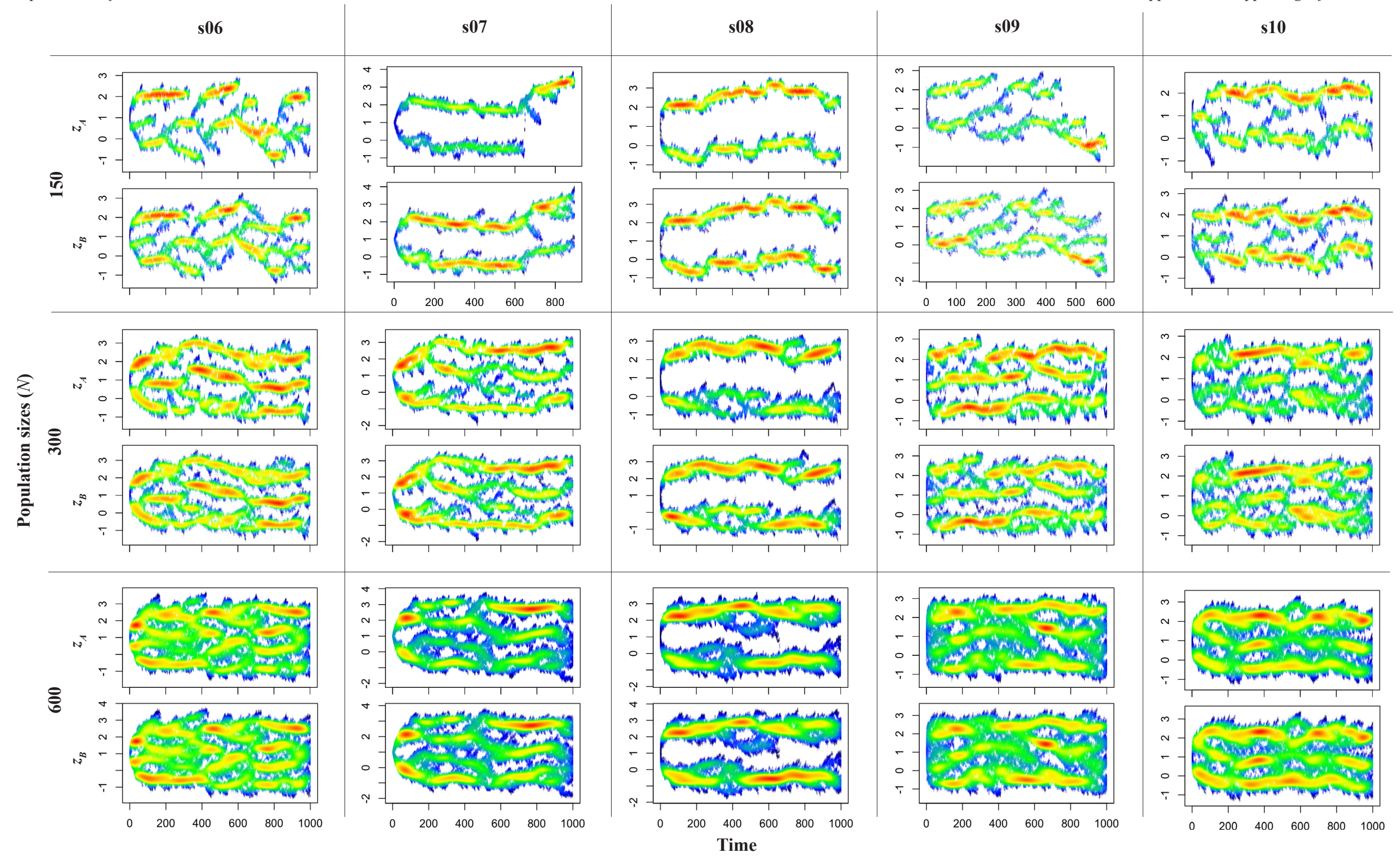

Figure A6-B. Phenotypic trajectories plots for traits $\mathrm{z}_{\mathrm{A}}$ and $\mathrm{z}_{\mathrm{B}}$ under parameter combinations (s06-s10) obtained using Latin Hypercube sampling. See Table 1 for descriptions of the parameters and their values. The columns present phenotypic trajectories under each parameter combination and rows show results for three population sizes $\left(N_{A}=N_{B}=150,300,600\right)$. Abscissae depict the number of time steps for which each simulation ran and follow the labels in the last row, ranging from 0 to 1000, except when indicated. Simulations with different duration ended due to species extinction. Colors represent density of individuals throughout the phenotypic space. $\omega_{B}=\lambda=1$. 


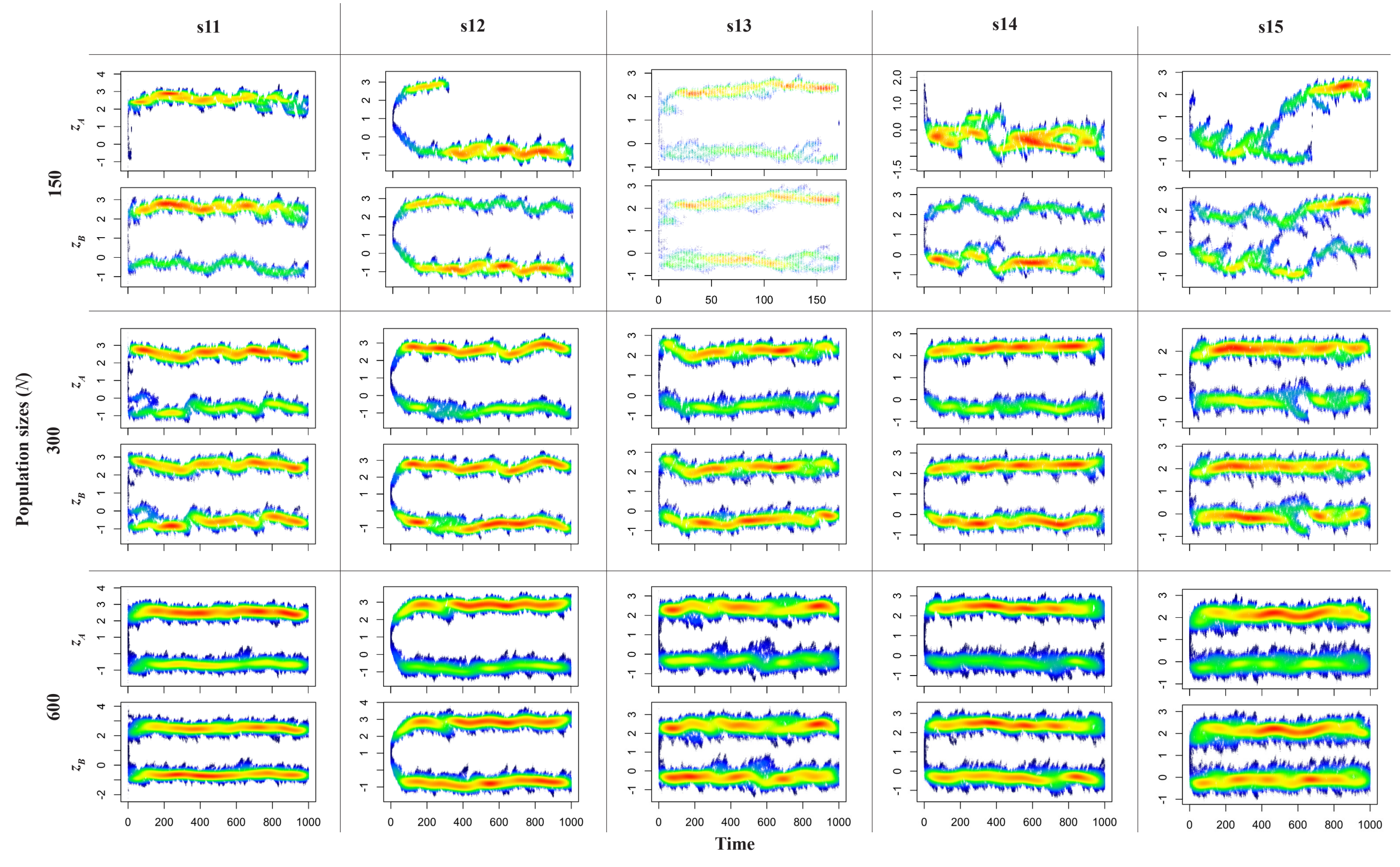

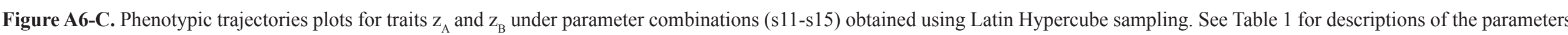

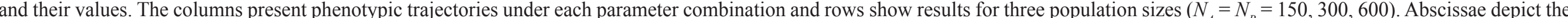

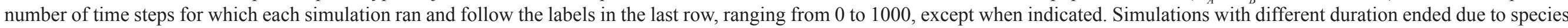
extinction. Colors represent density of individuals throughout the phenotypic space. $\omega_{B}=\lambda=1$. 


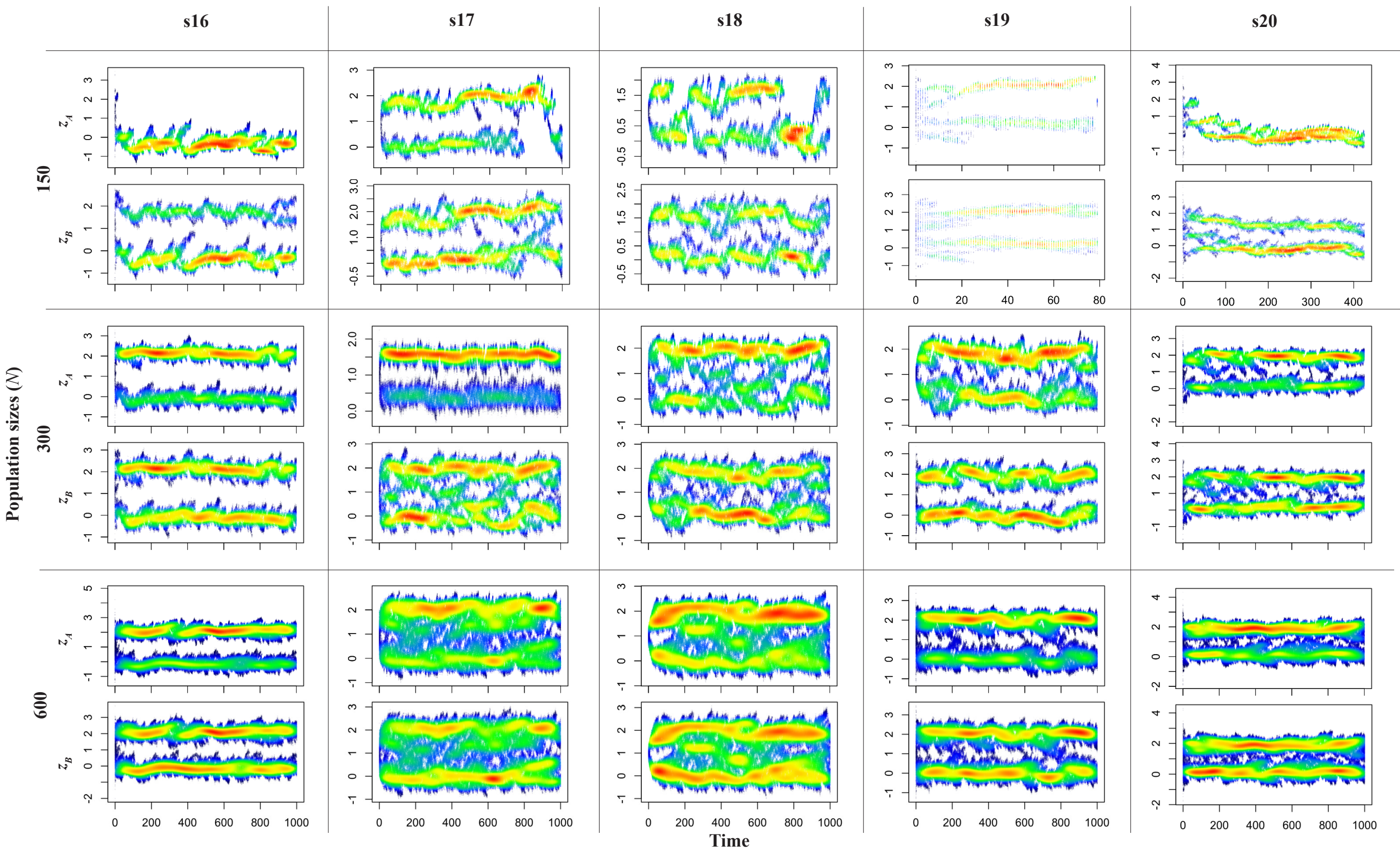

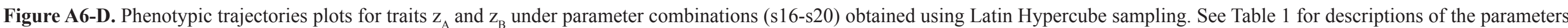
and their values. The columns present phenotypic trajectories under each parameter combination and rows show results for three population sizes $\left(N_{4}=N_{B}=150,300,600\right)$. Abscissae depict the number of time steps for which each simulation ran and follow the labels in the last row, ranging from 0 to 1000 , except when indicated. Simulations with different duration ended due to species extinction. Colors represent density of individuals throughout the phenotypic space. $\omega_{B}=\lambda=1$. 


\section{Symmetric constraint on the number of individual mutualistic partners}

In our simulation experiments, only individuals of species $B$ have a fixed number of mutualistic partners. Complementarily, we also explored an alternative scenario in which both species are constrained in relation to the number of partners with which individuals interact $\left(\omega_{A}=\omega_{B}\right)$. We defined a preference vector with mutualistic partners for each individual of species $B$ based on phenotype matching. Interaction occurred by rounds. In each round of interactions, the order of individuals of species $B$ choosing their preferred partner was randomly assigned. If a given individual of species $A$ reached $\omega_{A}$ interactions, it was removed from the mutualistic partner pool available for species $B$. In this scenario, in which individuals of both species have limits to the number of interaction events, the degree of diversification also drops following the increase in the value of $\omega_{A}=\omega_{B}$ (fig. A9).

\section{Relaxing the assumption of both species under disruptive selection}

We relaxed the assumption that both species are under disruptive selection. We ran 20 replicates for each combination of $\omega_{B}\left(\omega_{B}=8,64,512\right)$ and $\lambda$ values $(\lambda=0.1,1)$ for cases in which (i) only species $A$ is under disruptive selection, and (ii) only species $B$ experiences the disruptive regime.

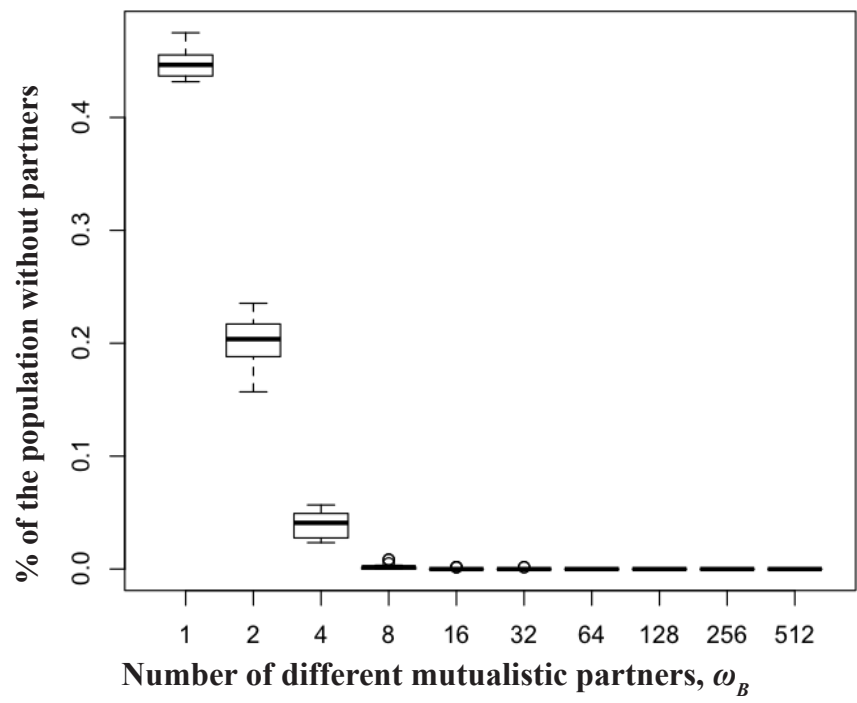

Figure A7. Percentage of the species $A$ population remaining with no mutualistic partner within the first generation, under different numbers of mutualistic partners $\omega_{B}$. Other parameters as in table A1 (s1).
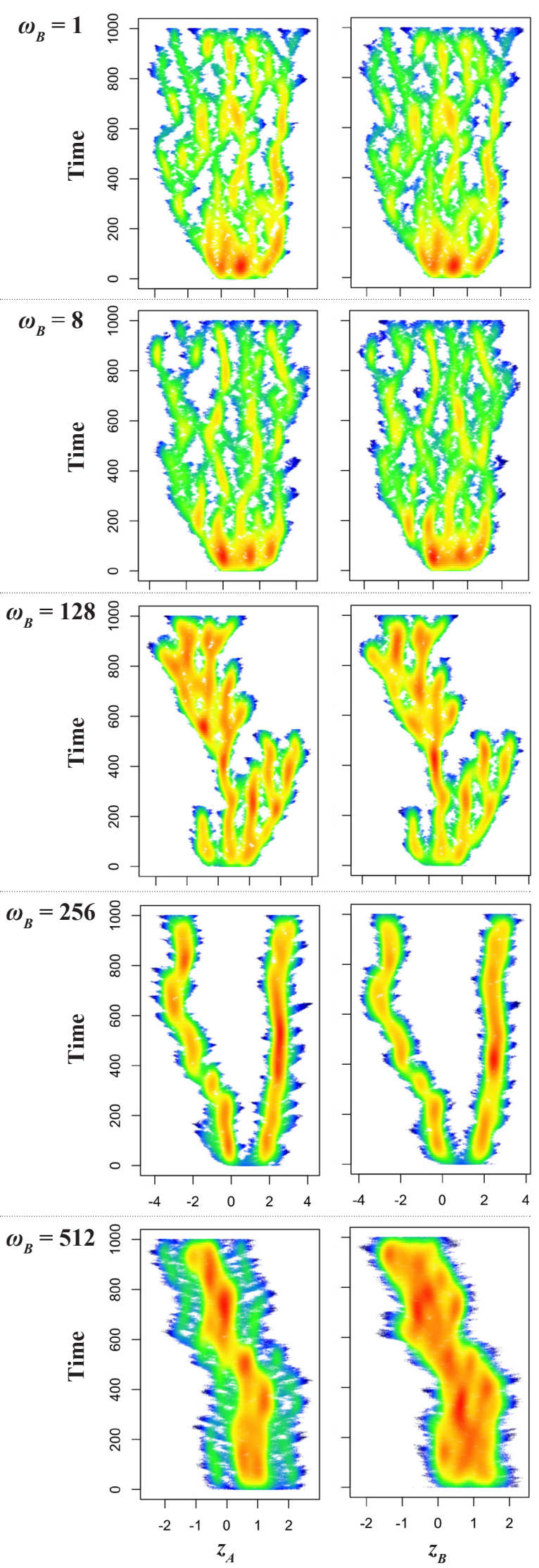

Figure A8. Individual-based simulations showing the effect of $\omega_{B}$ on adaptive diversification when $P_{m u t}$ is proportional to the mean trait complementarity of all interactions, instead of the sum of benefits obtained from each mutualistic partner (equation 4). Temperature colors represent density of individuals in the phenotypic space. Other parameters are the same as in table A1 (s1), with $N_{A}=N_{B}=600$. Abscissae values where $1 \leq \omega_{B} \leq 128$ are the same than those in the panel where $\omega_{B}=256$. 
Species richness is significantly lower (Tukey's HSD test, $Q=2.34, p<0.001$ ) in cases where species $A\left(S_{A}=3.88 \pm 2.23 \mathrm{spp}\right.$. and $\mathrm{SB}=4.00$ \pm 2.35 spp., $n=120$ simulations $)$ or species $B\left(S_{A}=\right.$ $1.28 \pm 0.58$ spp. and $S_{B}=1.19 \pm 0.41$ spp., $\mathrm{n}=120$ simulations) are not subject to disruptive selection compared to the simulations in which both species experience a disruptive selective regime $\left(S_{A}=5.18 \pm\right.$ $2.23 \mathrm{spp}$. and $S_{B}=5.3 \pm 2.3 \mathrm{spp} ., \mathrm{n}=120$ simulations, fig. A10). Despite such quantitative differences, similar effects of $\omega_{B}$ and $\lambda$ on diversification emerge if only species $B$ faces disruptive selection. However, diversification is severely constrained if only species $A$, the one with individuals being chosen within the mutualistic interaction, experiences the disruptive regime. In this case, a low degree of diversification occurs when the effect of mutualistic trait matching on fitness decreases (fig. A11). These results show that the degree of diversification achieved by $A$ is highly dependent on the selective regimes operating in its mutualistic partner, $B$.

\section{Relaxing the assumption of equal and constant population sizes}

We relaxed the assumption that the overall number of individuals within each species or group of species is equal and constant over time. A first scenario of stochastic population dynamics, which is presented in the main text, assumes that each species' population varies randomly around a mean. At each generation, each species population size is defined by the initial population size $\left(N_{A}=N_{B}=600\right)$ summed to a parameter $e$ whose value is sampled from a Gaussian with mean equal to zero and standard deviation $\sigma_{P(K)}$ (see the main text for details and results).

In a second simulation, we allowed more drastic stochastic fluctuations of population sizes. Departing from initial population sizes of $N_{O(A)}=N_{O(B)}$ $=600$, the number of individuals $N_{t(K)}$ of a species/ guild at the generation $t$ is given by $N_{t-1(K)}+\mathrm{V}$, where $V$ is a percentage of $N_{t-1(K)}$. At any given generation, $V$ is randomly assigned to be positive or negative.
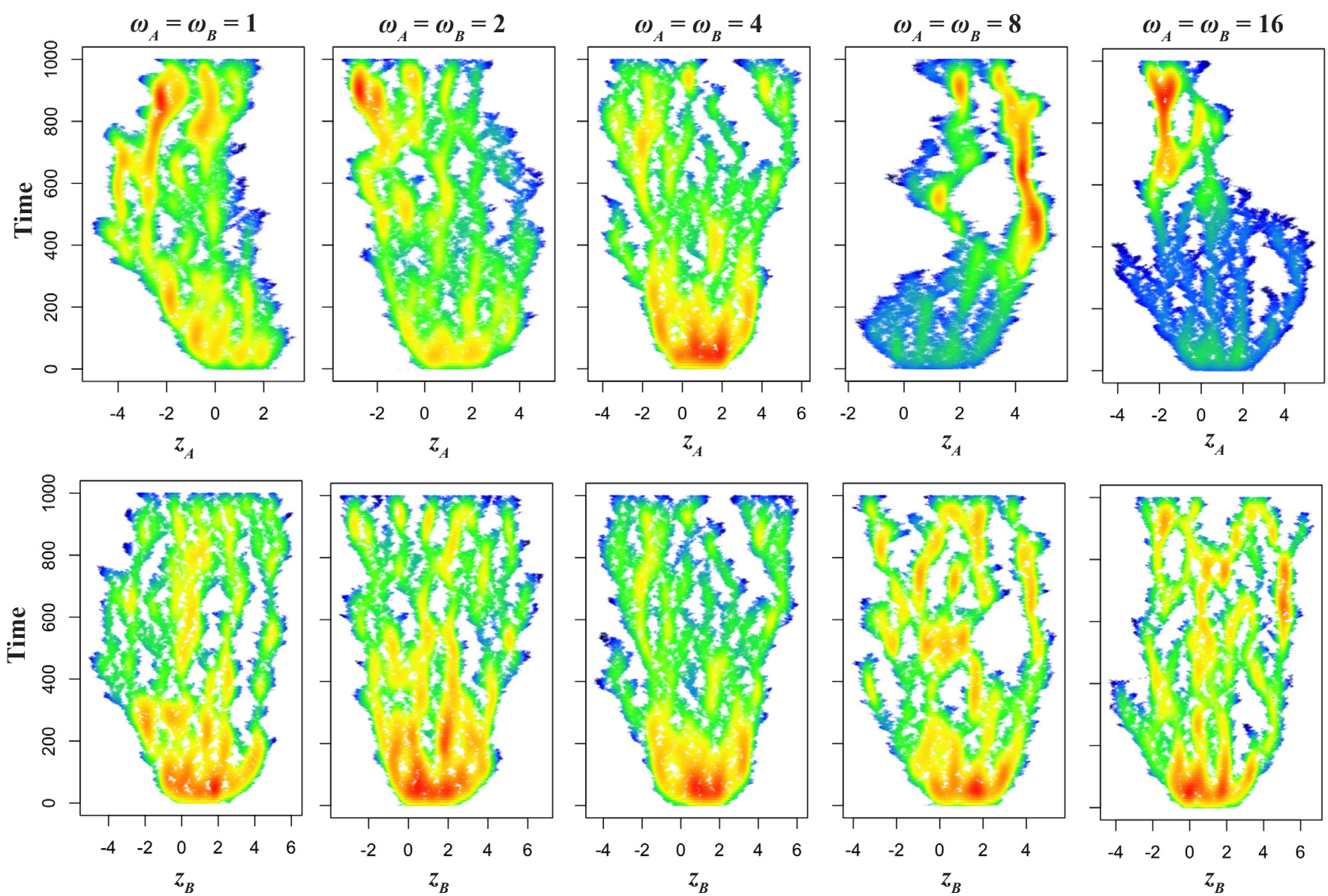

Figure A9. Adaptive diversification under increasing number of mutualistic partners per individual when both set of species have limits to the number of interaction events $\left(\omega_{A}=\omega_{B}\right)$. Interactions occur by rounds. The order of individuals of species $B$ choosing the partners is randomly assigned in each round. Each individuals interact $\omega_{K}$ times and than it is removed from the mutualistic pool. Colors represent individuals density. Other parameters are the same as in table A1 (s1), with $N_{A}=N_{B}=600$. 
We contrasted the diversification dynamics under different values of $\omega_{B}(8,32)$ and $V(1 \%, 5 \%)$. In the resulting population dynamics, population sizes vary independently and can assume values much lower or much higher than the original sizes. Despite such a wide variation in population size, the general trend of decreased diversification following increasing $\omega_{B}$ is also observed (Figure A12). The trend is robust even for higher values of $\omega_{B}(64,128)$ and $V(10 \%, 15 \%)$ (results not shown).

\section{Males under selective pressures arising from ecological interactions}

In our baseline model, only females have mating probabilities defined by two fitness components arising from ecological interactions, $P_{\text {comp }}$ and $P_{\text {mut }}$ (equations 3-5). Figure A13 shows the results of simulations in which males are also affected by the selective pressures generated by ecological interactions. We explored the effects of the number of different individual mutualistic partners, $\omega_{B}$, and of the impact of the degree of trait matching on fitness, $\lambda$, when the males mating pool is formed by a percentage $(50 \%$ or $25 \%)$ of the original males. The probability that a male will remain in the population to form the mating pool is computed following equations 3-5, but with $N_{A m}$ or $N_{K m}$, the number of males within the population of the species $A$ or $K$, replacing $N_{A f}$ or $N_{K f}$

We found that the effects of $\omega_{B}$ and $\lambda$ are qualitatively the same when males are also subject to selective pressures arising from ecological interactions. However, the overall species diversification decreases in the extreme case in which only $25 \%$ of the original males form the mating pool, probably due to an additional source of stabilizing selection imposed by the reduced availability of mating males.

\section{Species richness in natural mutualistic assemblages}

We compiled a dataset summarizing species richness in 57 natural assemblages to describe species richness in high-intimacy (high $\lambda$, low $\omega_{B}$ ) and lowintimacy (variable $\lambda$, high $\omega_{B}$ ) local mutualistic assemblages. We thus contrasted species richnessess between groups of mutualistic assemblages in which the interacting individuals have different degrees of biologicalintegration(Ollerton2006). Variabledegrees of interaction intimacy affect the organization of ecological interactions in different ways, with distinct consequences for the evolutionary and coevolutionary processes that shape species diversity (Guimarães et al. 2007, Fontaine et al. 2011, Pires and Guimarães

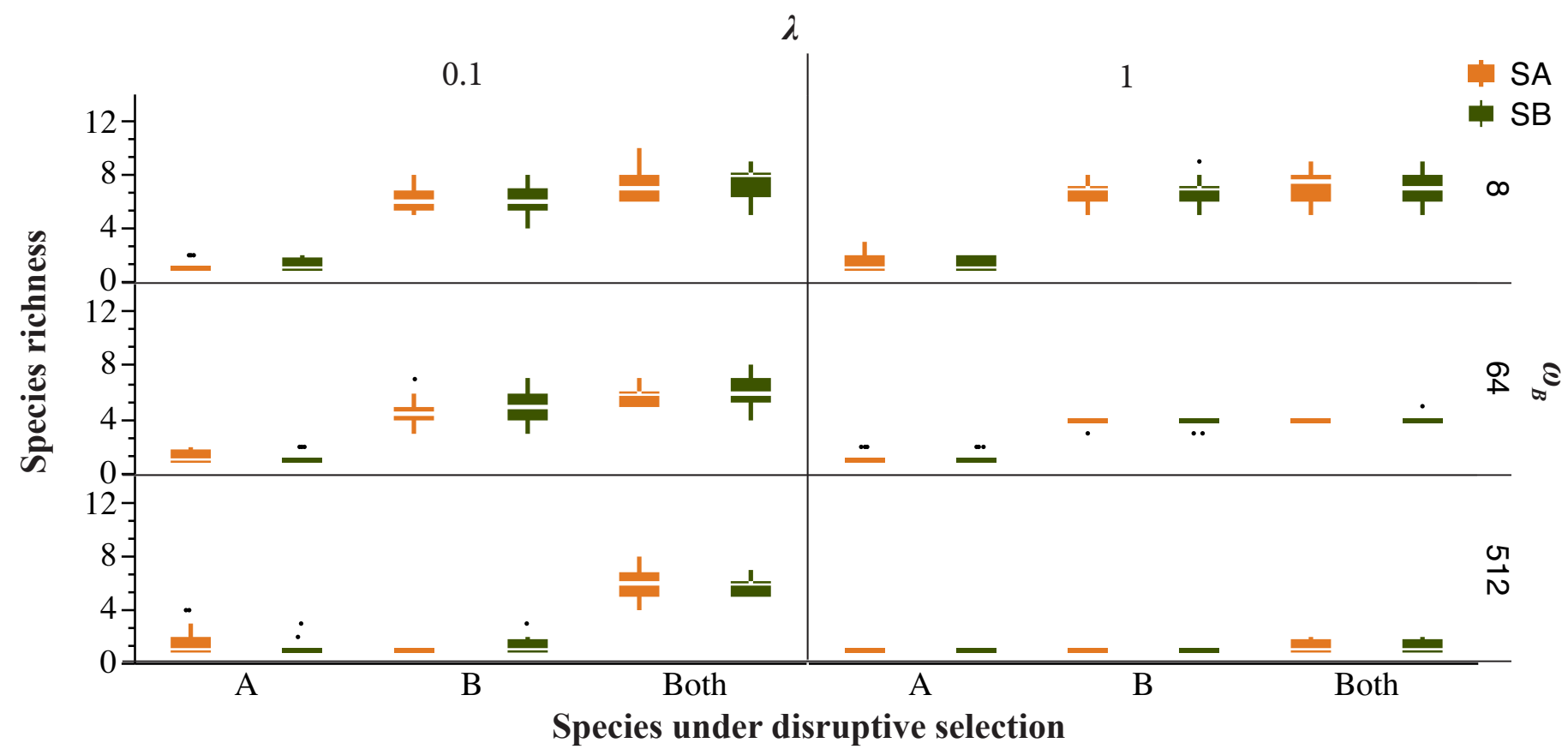

Figure A10. Asymptotic species richness for clades derived from species $A\left(S_{A}\right)$ and from species $B\left(S_{B}\right)$ when disruptive selection acts upon one of them $(A$ or $B)$ or both of them. Same parameters as in table A1 (s1), with $N_{A}=N_{B}=600$. 
2013). We obtained data from the Interaction Web Database website, available in the address http://www.nceas.ucsb.edu/interactionweb/, and from the literature. References are provided in Table A2.

Data on high-intimacy mutualisms totalize 35

Only species $A$ under disruptive selection

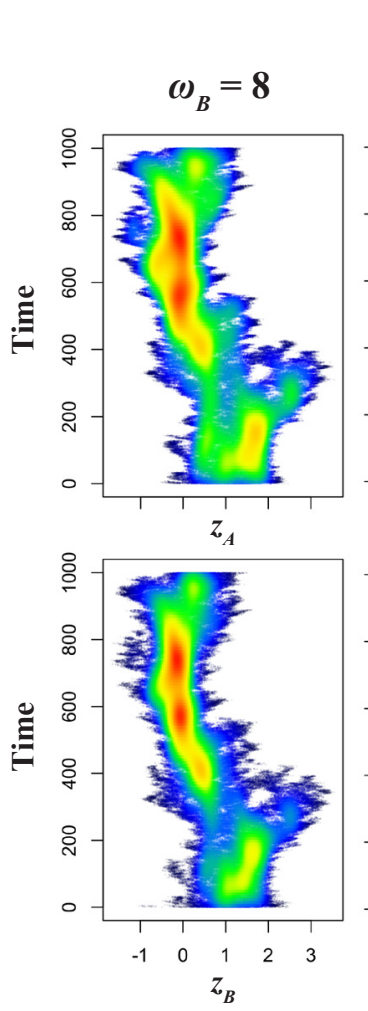

$$
\lambda=1
$$$$
\omega_{B}=128
$$
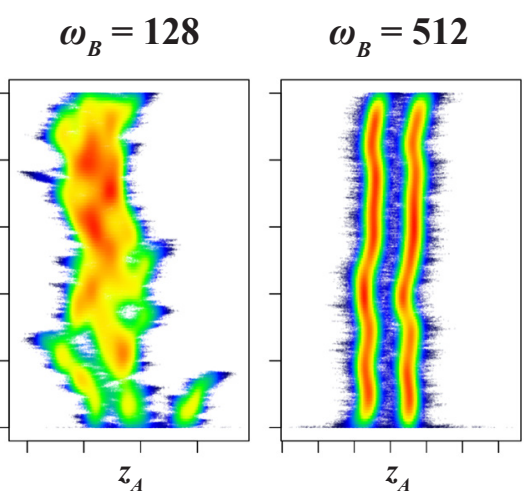

$z_{A}$
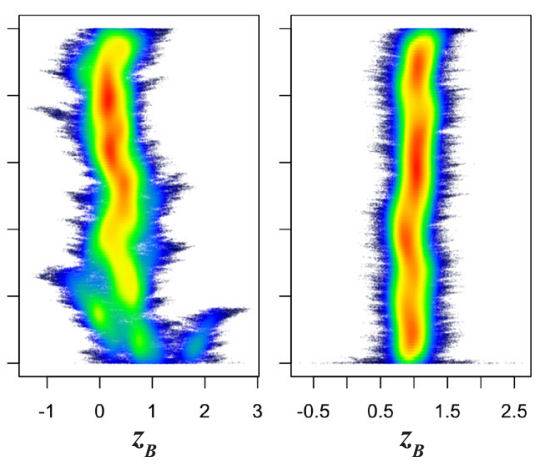

$$
\lambda=\mathbf{0 . 1}
$$

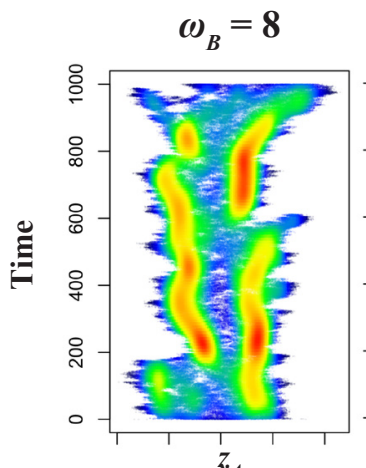

$\omega_{B}=128$
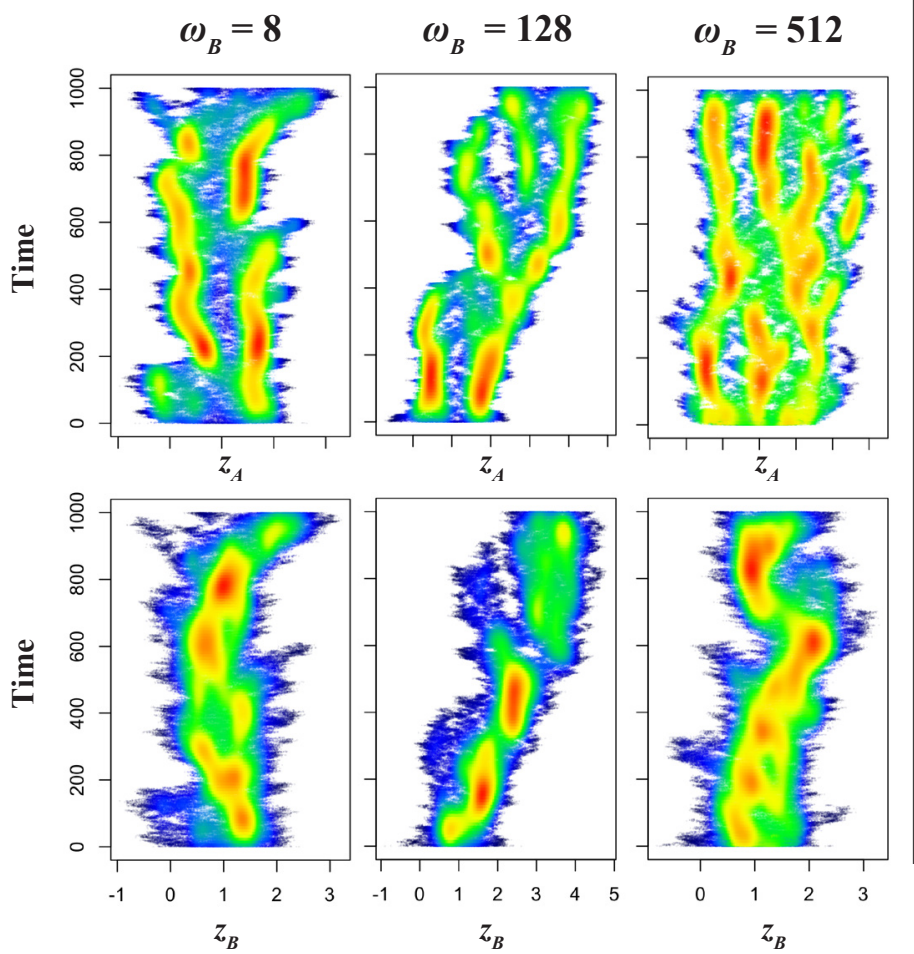

assemblages, including: obligate mutualisms between species of anemone fish and their host sea anemones (17 assemblages); intimate protection mutualisms between specialized ant species and myrmecophyte plants that provide housing and food to ant colonies ( 5

\section{Only species $B$ under disruptive selection}
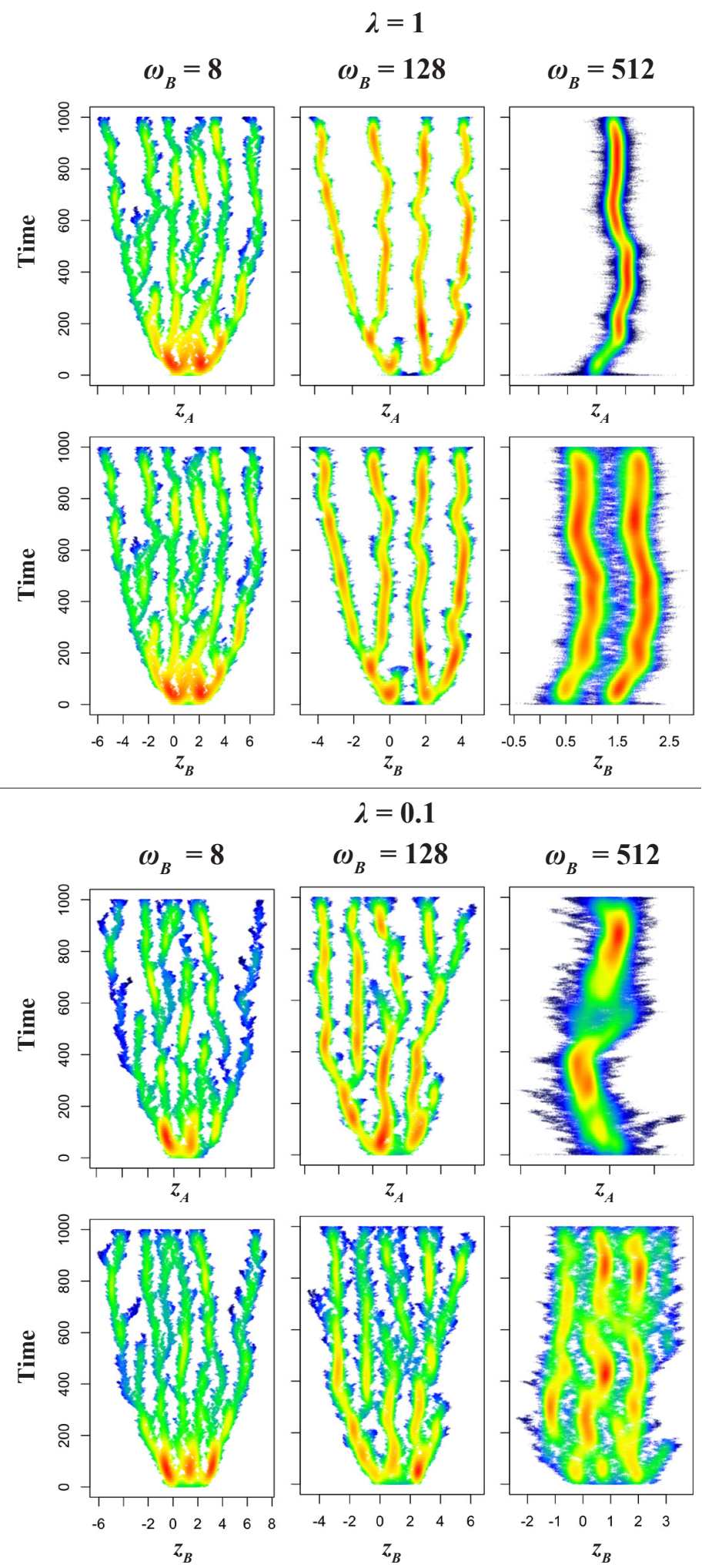

Figure A11. Phenotypic trajectories of traits $z_{A}$ and $z_{B}$ when only one of the species $(A$ or $B)$ is under disruptive selection. Same parameters as in table A1 (s1), with $N_{A}=N_{B}=600$. 
assemblages); highly specialized, obligate mutualisms between pollinating seed parasites and their host plant species (13 assemblages), encompassing interactions between figs and fig wasps (2 assemblages) and between Glochidion trees and Epicephala moths (11 assemblages).

Data depicting species richnessess in lowintimacy mutualisms totalize 22 assemblages, including: marine cleaning mutualisms involving cleaner species of shrimps or fishes removing ectoparasites and other material from their client fishes (3 assemblages); generalized ant-plant mutualisms, including ant-mediated seed dispersal (2 assemblages) and generalized ant-plant protection mutualisms ( 3 assemblages); and mutualisms between animal pollinators and flowering plant species (14 assemblages).
High-intimacy mutualistic assemblages are relatively species-poor ( $S=10.7 \pm 8.3$ species), with assemblages of anemones and anemone fishes and of plants and pollinating seed parasite holding the lowest species numbers. On the other hand, low-intimacy mutualisms have up to one order of magnitude more species $(S=97.2 \pm 47.4$ species, $t=11.5, P<0.0001$ ), with plant-pollinator assemblages representing the richest systems (fig. 4, main text).

$V=1 \%$
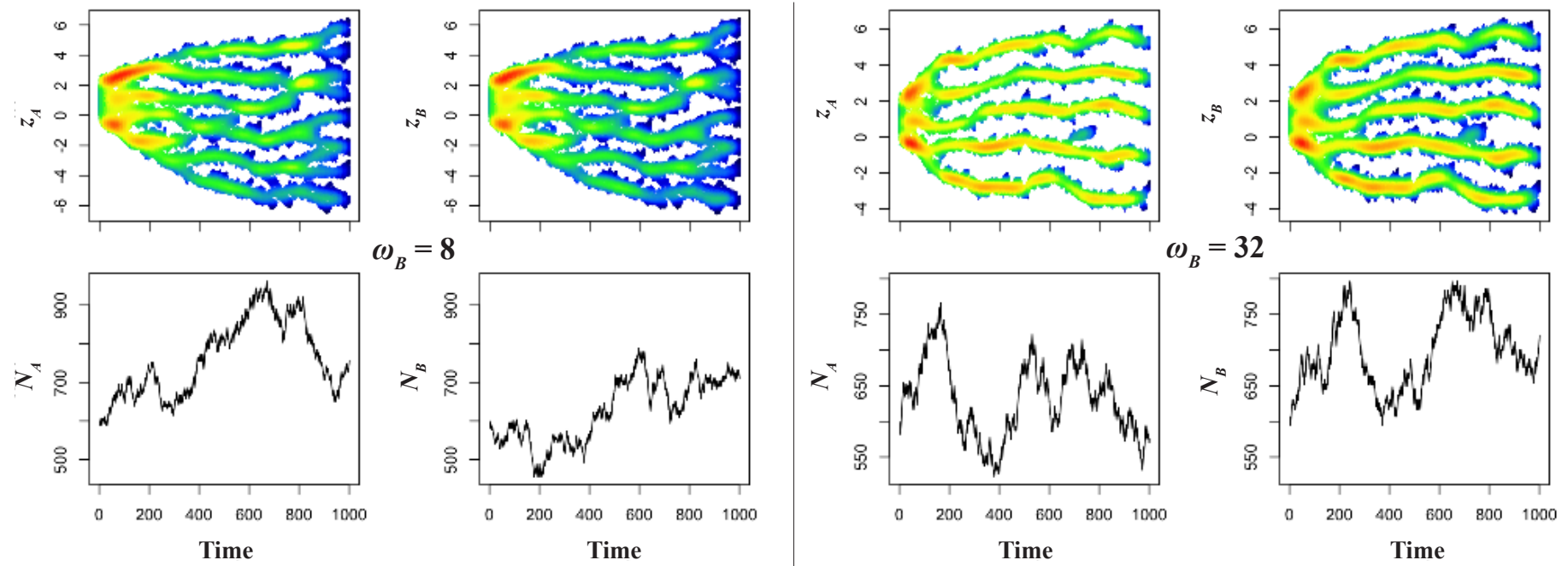

$V=5 \%$
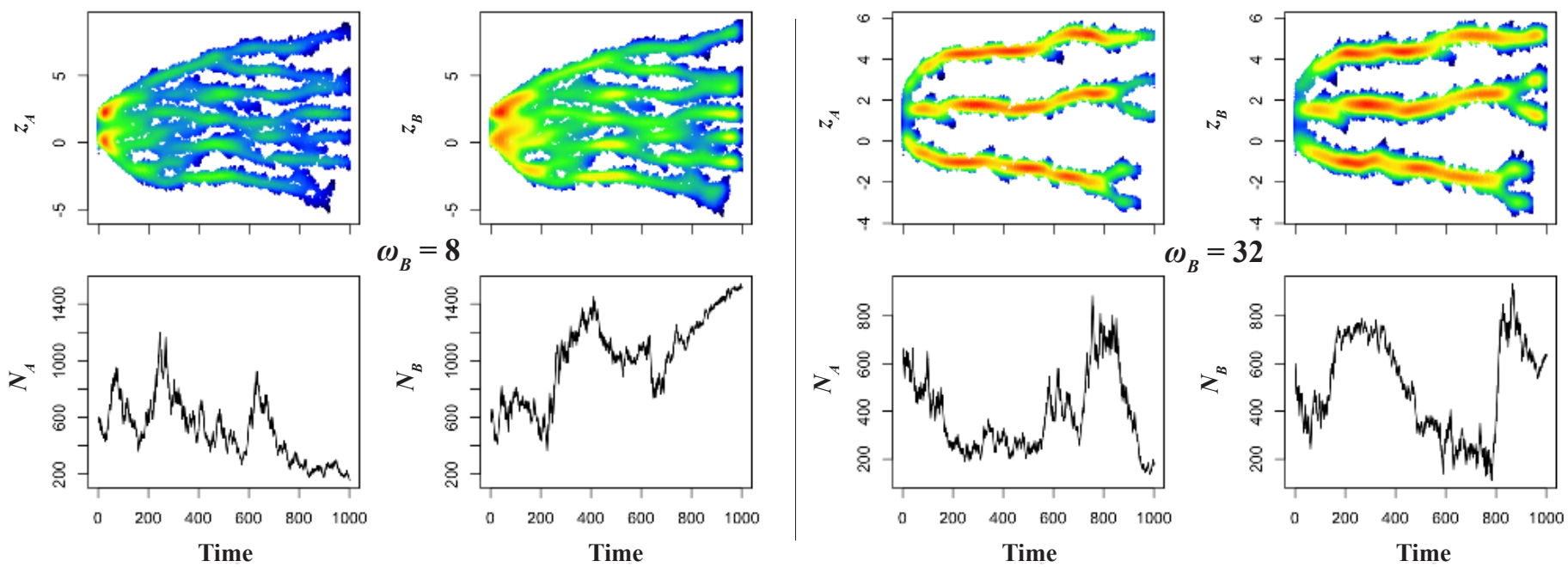

$\omega_{B}=32$

Figure A12. Phenotypic trajectories of traits $z_{A}$ and $z_{B}$ when population sizes vary stochastically. $\omega_{B}$ is the number of different mutualistic partners of each individual of species B. $V$ is the parameter defining the degree of stochasticity to which the populations are subject. At any given generation, $V$ is randomly assigned to be positive or negative. After that, a percentage $V$ of the previous generation size is summed or subtracted to define the current generation size. 


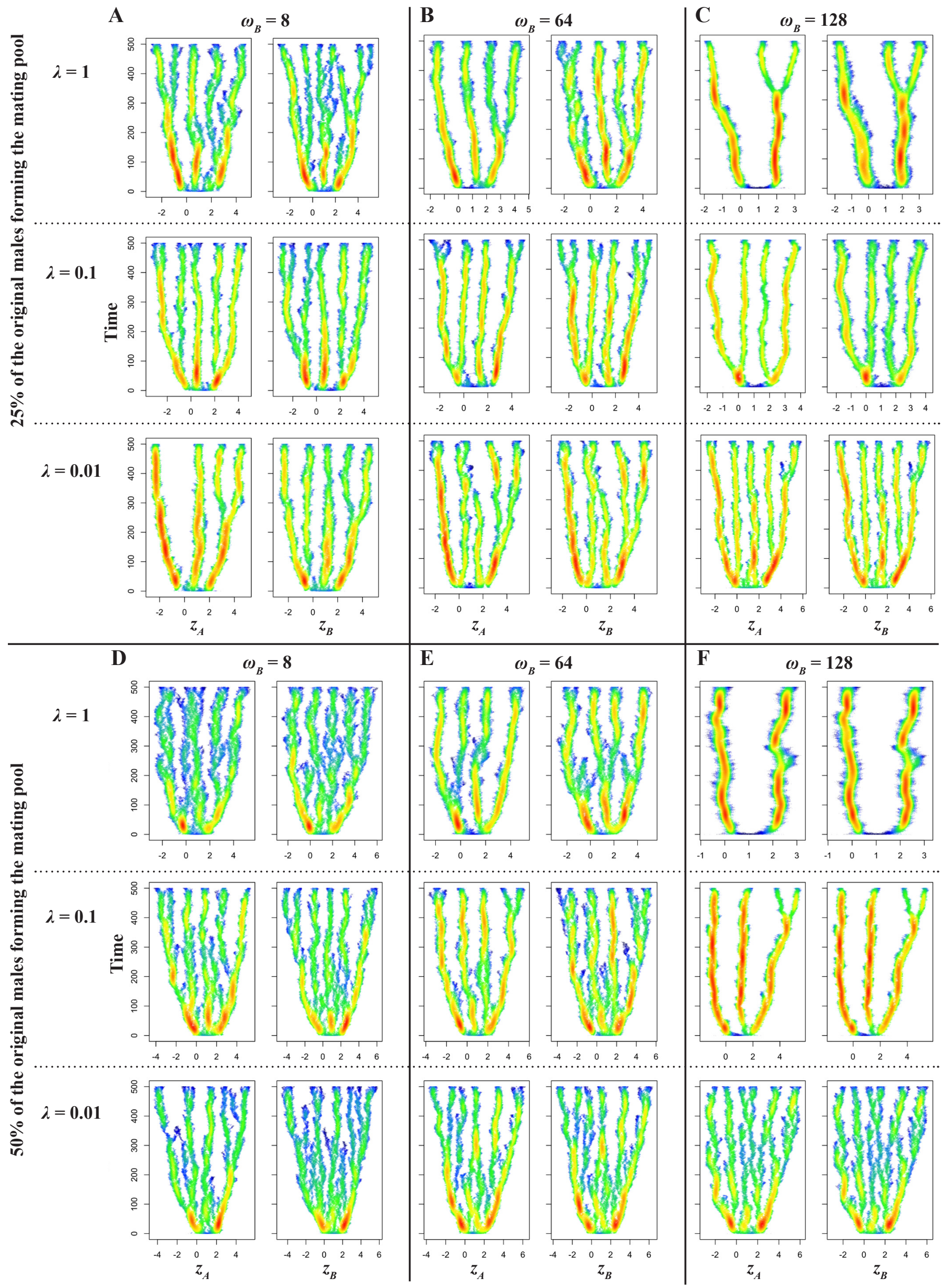

Figure A13. Species diversification when males are also under selective pressures arising from ecological interactions. The effects of $\omega_{B}$ and and $\lambda$ on species diversification are qualitatively the same than observed in Experiment 2. (A-C) The male mating pool is formed by a quarter of the original male subpopulation. (D-F) The male mating pool is formed by half of the original male subpopulation. The probability that a male remains in the population to form the mating pool is defined by the fitness components arising from ecological interactions (see the text for details). 
Table A2. Information on the datasets used to describe species richeness in natural mutualistic assemblages.

\begin{tabular}{|c|c|c|c|}
\hline $\begin{array}{l}\text { Interaction } \\
\text { Intimacy }\end{array}$ & Type of Mutualism & Number of datasets & References \\
\hline High & $\begin{array}{l}\text { Obligate mutualism } \\
\text { between anemone fishes and their } \\
\text { host sea anemones }\end{array}$ & 17 & $\begin{array}{l}\text { Frisch and Hobbs 2009; Ricciardi et al. } \\
2010\end{array}$ \\
\hline High & $\begin{array}{l}\text { Mutualisms between } \\
\text { specialized ants and myrmecophyte } \\
\text { plants hosting their nests }\end{array}$ & 5 & $\begin{array}{l}\text { Davidson et al. 1989; Davidson and } \\
\text { Fisher 1991; Fonseca and Ganade 1996; } \\
\text { Guimarães et al. } 2007\end{array}$ \\
\hline High & $\begin{array}{l}\text { Highly specialized, obligate } \\
\text { mutualisms between plants and } \\
\text { pollinating seed parasites }\end{array}$ & 13 & $\begin{array}{l}\text { Machado et al. 2005; Kawakita and Kato } \\
\text { 2006; Azuma et al. 2010; Hembry 2012; } \\
\text { Hembry et al. } 2013\end{array}$ \\
\hline Low & $\begin{array}{l}\text { Cleaning mutualisms involving } \\
\text { cleaner species (shrimps or fishes) } \\
\text { and their client species (fishes) }\end{array}$ & 3 & $\begin{array}{l}\text { Johnson and Ruben 1988, Wicksten } \\
\text { 1998; Sazima } 2002\end{array}$ \\
\hline Low & Ant-mediated seed dispersal & 2 & $\begin{array}{l}\text { Pizo and Oliveira 2000; Passos and } \\
\text { Oliveira } 2003\end{array}$ \\
\hline Low & $\begin{array}{l}\text { Generalized ant-plant protection } \\
\text { mutualisms }\end{array}$ & 3 & $\begin{array}{l}\text { Rico-Gray 1993; Rico-Gray et al. 1998; } \\
\text { Diaz-Castelazo et al. } 2004\end{array}$ \\
\hline Low & $\begin{array}{l}\text { Mutualisms between animal } \\
\text { pollinators and flowering plants }\end{array}$ & 14 & $\begin{array}{l}\text { Hocking 1968; Kevan 1970; } \\
\text { Arroyo et al. 1982; Barrett and } \\
\text { Helenurm 1987; Inouye and Pyke 1988; } \\
\text { Elberling and Olesen 1999; Memmott } \\
\text { 1999, Medan et al. 2002; Vázquez and } \\
\text { Simberloff 2002, 2003; Kaiser-Bunbury } \\
\text { et al. } 2010\end{array}$ \\
\hline
\end{tabular}

\section{Literature cited in the Online Appendix}

Arroyo, M. T. K., R Primack, and J. Armesto. 1982. Community studies in pollination ecology in the high temperate Andes of central Chile. I. Pollination mechanisms and altitudinal variation. American Journal of Botany 69: 82-97.

Azuma, H., R. D. Harrison, K. Nakamura, and Z. H. Su. 2010. Molecular phylogenies of figs and fig-pollinating wasps in the Ryukyu and Bonin (Ogasawara) islands, Japan. Genes \& Genetic Systems 85: 177-192.

Barrett, S. C. H., and K. Helenurm. 1987. The reproductive biology of boreal forest herbs. I. Breeding systems and pollination. Canadian Journal of Botany 65: 2036-2046.

Carnell, R. 2009. Lhs: Latin Hypercube Samples. R package version 0.5 .

Davidson, D. W., R. R. Snelling, and J. T. Longino. 1989. Competition among ants for myrmecophytes and the significance of plant trichomes. Biotropica 21: 6473.

Davidson, D. W., and B. L. Fisher. 1991. Symbiosis of ants with Cecropia as a function of light regime. Pages 289309 in C. R. Huxley and D. F. Cutler (eds). Ant-plant interactions. Oxford University Press, Oxford.
Diaz-Castelazo, C., V. Rico-Gray, P. S. Oliveira, and M. Cuautle. 2004. Extrafloral nectary-mediated ant-plant interactions in the coastal vegetation of Veracruz, Mexico: richness, occurrence, seasonality, and ant foraging patterns. Ecoscience 11: 472-481.

Doebeli, M., and U. Dieckmann. 2000. Evolutionary branching and sympatric speciation caused by different types of ecological interactions. The American Naturalist 156: S77-S101.

Dupont, Y. L., and D. M. Hansen. 2003. Structure of a plant-flower-visitor network in the high-altitude subalpine desert of Tenerife, Canary Islands. Ecography 26: 301-310.

Elberling, H., and J. M. Olesen. 1999. The structure of a high latitude plant-flower visitor system: the dominance of flies. Ecography 22: 314-323.

Fonseca, C. R., and G. Ganade. 1996. Asymmetries, compartments and null interactions in an Amazonian ant-plant community. Journal of Animal Ecology 65: 339-347.

Fontaine, C., P. R. Guimarães Jr, S. Kéfi, N. Loeuille, J. J. Memmott, W. H. van der Putten, F. J. F. van Veen, and E. Thébault. 2011. The ecological and evolutionary implications of merging different types of networks. 
Ecology Letters 14: 1170-1181.

Frisch, A. J., and J-P. A. Hobbs. 2009. Rapid assessment of anemone and anemonefish populations at the Keppel Islands [electronic resource]: a report to the Great Barrier Reef Marine Park Authority. Research Publication no. 94, Great Barrier Reef Marine Park Authority, Commonwealth of Australia.

Guimarães, P. R, Jr, V. Rico-Gray, P. S. Oliveira, T. J. Izzo, S. F. Reis, and J. N. Thompson. 2007. Interaction intimacy affects structure and coevolutionary dynamics in mutualistic networks. Current Biology 17: 17971803.

Hembry, D. H. 2012. Coevolutionary diversification of leafflower moths (Lepidoptera: Gracillariidae:

Epicephala) and leafflower trees (Phyllanthaceae: Phyllanthus sensu lato [Glochidion]) in Southeastern Polynesia. PhD Thesis. University Of California, Berkeley.

Hembry, D. H., A. Kawakita, N.E. Gurr, M.A. Schmaedick, B.G. Baldwin, and R.G. Gillespie. 2013. Non-congruent colonizations and diversification in a coevolving pollination mutualism on oceanic islands. Proceedings of the Royal Society B 280: 20130361

Hocking, B. 1968. Insect-flower associations in the high Arctic with special reference to nectar. Oikos 19:359388.

Inouye, D. W., and G. H. Pyke. 1988. Pollination biology in the snowy mountains of Australia - comparisons with Montane Colorado, USA. Australian Journal of Ecology 13: 191-210.

Johnson, W. S. \& Ruben, P. 1988 Cleaning behavior of Bodianus rufus, Thalassoma bifasciatum, Gobiosoma evelynae, and Periclimenes pedersoni along a depth gradient at Salt River Submarine Canyon, St. Croix. Environmental Biology of Fishes 23: 225-232.

Kaiser-Bunbury, C. N., S. Muff, J. J. Memmott, C. B. Mueller, and A. Caflisch. 2010. The robustness of pollination networks to the loss of species and interactions: a quantitative approach incorporating pollinator behaviour. Ecology Letters 13: 442-452.

Kawakita, A., and M. Kato. 2006. Assessment of the diversity and species specificity of the mutualistic association between Epicephala moths and Glochidion trees. Molecular Ecology 15: 3567-3581.

Kevan, P. G. 1970. High Arctic insect-flower visitor relations: the inter-relationships of arthropods and flowers at Lake Hazen, Ellesmere Island, Northwest Territories, Canada. PhD Thesis. University of Alberta, Canada.

Machado, C. A., N. Robbins, M. T. P. Gilbert, and E. A. Herre. 2005. Critical review of host specificity and its coevolutionary implications in the fig/fig-wasp mutualism. Proceedings of the National Academy of Sciences of the USA 102: 6558-6565.
Medan, D, N. H. Montaldo, M. Devoto, A. Mantese, V. Vasellati, and N. H. Bartoloni. 2002. Plant-pollinator relationships at two altitudes in the Andes of Mendoza, Argentina. Arctic, Antarctic and Alpine Research 34: 233-241.

Memmott, J. 1999. The structure of a plant-pollinator food web. Ecology Letters 2: 276-280.

Ollerton, J. 2006. "Biological Barter": patterns of specialization compared across different mutualisms. Pages 411-435 in N. M. Waser and J. Ollerton (eds). Plant-pollinator interactions: from specialization to generalization. University of Chicago Press, Chicago.

Passos, L., and P.S. Oliveira. 2003. Interactions between ants, fruits and seeds in a Restinga Forest in SouthEastern Brazil. Journal of Tropical Ecology 19: 261270.

Pires, M. M., and P. R. Guimarães Jr. 2013. Interaction intimacy organizes networks of antagonistic interactions in different ways. Journal of The Royal Society Interface, doi: 10.1098/rsif.2012.0649.

Pizo, M. A., and P. S. Oliveira. 2000. The use of fruits and seeds by ants in the Atlantic Forest of Southeast Brazil. Biotropica 32: 851-861.

Ricciardi,F., Massimo B., and J. Ollerton. 2010. Assemblage and interaction structure of the anemonefish-anemone mutualism across the Manado Region of Sulawesi, Indonesia. Environmental Biology of Fishes 87: 333347.

Rico-Gray, V. 1993. Use of plant-derived food resources by ants in the dry tropical lowlands of Coastal Veracruz, Mexico. Biotropica 25: 301-315.

Rico-Gray, V., M. Palacios-Rios, J. G. Garcia-Franco, and W. P. Mackay. 1998. Richness and seasonal variation of ant-plant associations mediated by plant-derived food resources in the semiarid Zapotitlán Valley, México. American Midland Naturalist 140: 21-26.

Sazima, C. 2002. Atividade de limpeza de duas espécies sintópicas de peixes limpadores e diversidade de seus clientes em Abrolhos, Bahia. MSc. Thesis, Universidade Estadual Paulista, UNESP, Rio Claro, Brazil.

Stein, M. 1987. Large sample properties of simulations using Latin Hypercube Sampling. Technometrics 29: 143-151.

Vázquez, D. P, and D. Simberloff. 2002. Ecological specialization and susceptibility to disturbance: conjectures and refutations. The American Naturalist 159: 606-623.

Vázquez, D. P, and D Simberloff. 2003. Changes in interaction biodiversity induced by an introduced ungulate. Ecology Letters 6: 1077-1083.

Wicksten, M. K. 1998. Behaviour of cleaners and their client fishes at Bonaire, Netherlands Antilles. Journal of Natural History 32, 13-30. 